\title{
Existence of Positive Solutions and Asymptotic Behavior for Evolutionary $q(x)$-Laplacian Equations
}

\author{
Aboubacar Marcos $(\mathbb{D}$ and Ambroise Soglo \\ Institut de Mathématiques et de Sciences Physiques, Université d'Abomey-Calavi, Cotonou, Benin \\ Correspondence should be addressed to Ambroise Soglo; ambroise.soglo@yahoo.fr
}

Received 21 January 2020; Revised 25 March 2020; Accepted 5 June 2020; Published 25 July 2020

Academic Editor: Jianquan Lu

Copyright ( 2020 Aboubacar Marcos and Ambroise Soglo. This is an open access article distributed under the Creative Commons Attribution License, which permits unrestricted use, distribution, and reproduction in any medium, provided the original work is properly cited.

In this paper, we extend the variational method of M. Agueh to a large class of parabolic equations involving $q(x)$-Laplacian parabolic equation $(\partial \rho(t, x) / z t)=\operatorname{div}_{x}\left(\rho(t, x)\left|\nabla_{x}\left(G^{\prime}(\rho)+V\right)\right|{ }^{q(x)-2} \nabla_{x}\left(G^{\prime}(\rho)+V\right)\right)$. The potential $V$ is not necessarily smooth but belongs to a Sobolev space $W^{1, \infty}(\Omega)$. Given the initial datum $\rho_{0}$ as a probability density on $\Omega$, we use a descent algorithm in the probability space to discretize the $q(x)$-Laplacian parabolic equation in time. Then, we use compact embedding $W^{1, q(.)}(\Omega) \hookrightarrow \hookrightarrow L^{q(.)}(\Omega)$ established by Fan and Zhao to study the convergence of our algorithm to a weak solution of the $q(x)-$ Laplacian parabolic equation. Finally, we establish the convergence of solutions of the $q(x)$-Laplacian parabolic equation to equilibrium in the $p($.$) -variable exponent Wasserstein space.$

\section{Introduction and the Main Results}

In this paper, we study the existence of positive solutions and the asymptotic behavior for a class of parabolic equations involving parabolic equations governed by the $q(x)$-Laplacian operator:

$$
\begin{array}{r}
\frac{\partial \rho(t, x)}{\partial t}=\operatorname{div}_{x}\left(\rho(t, x)\left|\nabla_{x}\left(G^{\prime}(\rho)+V\right)\right|^{q(x)-2} \nabla_{x}\left(G^{\prime}(\rho)+V\right)\right), \\
\text { in }[0,+\infty[\times \Omega,
\end{array}
$$

where $\Omega \subset \mathbb{R}^{d}$ is a convex, bounded, and smooth domain of $\mathbb{R}^{d}, G:[0,+\infty[\longrightarrow \mathbb{R}$ is a convex function of class $\left.\left.C^{2}(] 0, \infty[), q():. \Omega \longrightarrow\right] 1,+\infty\right]$ is a continuous function, and $V: \Omega \longrightarrow \mathbb{R}$ belongs to a Sobolev space $W^{1, \infty}(\Omega)$.

$q(x)$-Laplacian parabolic equation type is a broad family of parabolic equations including many equations emerging in the mathematical modeling of a variety of phenomena in physics such as the flow of compressible fluids in nonhomogeneous isotropic porous media, the behavior of electrorheological fluids [1,2], image processing [3], and the curl systems emanating from electromagnetism $[4,5]$.
Some authors have studied the existence of solutions of the $q(x)$-Laplacian parabolic equation with the variable exponent, when $G(t)=t^{2}$ and $V \equiv 0$ (see $[1,2,6]$ ), for a given initial datum $\rho_{0}$ and a homogeneous boundary condition. In their works, they use an approximation method to approach the $q(x)$-Laplacian parabolic equation by regularized problems under the following conditions: $\rho_{0} \in L^{\infty}(\Omega)$ and $\left|\nabla_{x} \rho_{0}\right|^{q_{+}} \in L^{1}(\Omega)$.

In [7], M. Agueh studied the existence of positive solutions for the $q(x)$-Laplacian parabolic equation when the variable exponent $q(x) \equiv q$ is constant (with $q>1$ ), and the potential $V$ is a convex function of class $C^{1}(\Omega)$. Moreover, the author in [7] proved that the parabolic $q$-Laplacian equation

$$
\begin{array}{r}
\frac{\partial \rho(t, x)}{\partial t}=\operatorname{div}_{x}\left(\rho(t, x)\left|\nabla_{x}\left(G^{\prime}(\rho)+V\right)\right|{ }^{q-2} \nabla_{x}\left(G^{\prime}(\rho)+V\right)\right), \\
\text { in }[0,+\infty[\times \Omega,
\end{array}
$$

is a gradient flow of the functional $E(\rho)=\int_{\Omega}(G(\rho)+\rho V) \mathrm{d} x$ with respect to the $p$-Wasserstein distance $(p=(q / q-1))$ defined by 


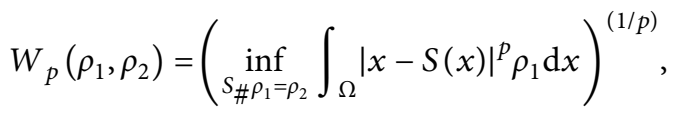

where $\rho_{1}$ and $\rho_{2}$ are two probability densities on $\Omega$.

In fact, by fixing the time step $h>0$ and a probability density $\rho_{0}$ on $\Omega$, the author defines $\rho_{k-1}\left(k \in \mathbb{N}^{*}\right)$ as the solution of (2) at $t=h(k-1)$ and $\rho_{k}$ as the solution of (2) at $t=h k$ such that $\rho_{k}$ is the unique solution of the variational problem

$$
\left(P_{k}\right): \inf _{\rho \in P(\Omega)}\left[E(\rho)+\frac{1}{\mathrm{ph}^{p-1}} W_{p}^{p}\left(\rho, \rho_{k-1}\right)\right]
$$

Then, the author established the convergence of the approximate solutions $\left(\rho_{k}\right)_{k}$ to a weak solution of the Laplacian parabolic equation . Here, we extend the work of [7] to a general case where $V \neq 0$ may not be smooth but belongs to a Sobolev space $W^{1, \infty}(\Omega)$. Roughly speaking, we use mass the transportation method borrowing ideas from $[7,8]$ to establish the existence of positive solutions and the long time behavior of solutions of the Laplacian parabolic equation. As in [7], we prove that the Laplacian parabolic equation is a gradient flow of the functional $E(\rho)=\int_{\Omega}[G(\rho)+\rho V] \mathrm{d} x$ with respect to the $p()-$. Wasserstein distance $W_{p(.)}$ defined by

$$
W_{p(.)}\left(\rho_{1}, \rho_{2}\right)=\inf _{\rho(t, .)}\left\{\|v\|_{L_{\rho(t .)}^{p(.)}([0,1] \times \Omega)}, \quad \frac{\partial \rho(t, x)}{\partial t}+\operatorname{div}_{x}(\rho(t, x) v(t, x))=0, \quad \text { in } \Omega, \rho(0, .)=\rho_{1} \text { and } \rho(1, .)=\rho_{2}\right\} .
$$

Next, we proceed with the discretization of the $q(x)$ Laplacian parabolic equation as follows: fixing the time step $h>0$ and a probability density $\rho_{0}$ on $\Omega$, we define $\rho_{k}$ as the approximate solution of the $q(x)$-Laplacian parabolic equation at $t_{k}=h k$, which minimizes the variational problem

$$
\left(P_{k}\right): \inf _{\rho \in P(\Omega)}\left\{\int_{\Omega}[G(\rho)+\rho V] \mathrm{d} x+K_{p(.)}^{h}\left(\rho_{k-1}, \rho\right)\right\}
$$

where

$$
K_{p(.)}^{h}\left(\rho_{k-1}, \rho\right)=\inf _{\gamma \in \Pi\left(\rho_{k-1}, \rho\right)} \int_{\Omega \times \Omega} \frac{|x-y|^{p(x)}}{p(x) h^{p(x)-1}} \mathrm{~d} \gamma .
$$

Here, $\Pi\left(\rho_{k-1}, \rho\right)$ is the set of all probability measures on $\Omega \times \Omega$ having $\rho_{k-1}$ and $\rho$ as their marginals, and

$$
K_{p(.)}^{h}\left(\rho_{k-1}, \rho\right), \quad k \geq 1,
$$

is the Monge-Kantorovich work associated to the cost $c_{h}(x, y)=\left(|x-y|^{p(x)} / p(x) h^{p(x)-1}\right)$.

The establishment of our result will be derived according to the following steps:

(1) Given $\rho_{0}$ as a probability density on $\Omega$ such as $N \leq \rho_{0} \leq M$ and $V \in L^{\infty}(\Omega)$, we prove that $\left(P_{k}\right)$ admits a unique solution $\rho_{k}$ which satisfies $N \leq \rho_{0} \leq M$ (see Lemma 1$)$.

(2) We prove in (28) that the Kantorovich problem

$$
(K): \inf _{\gamma \in \Pi\left(\rho_{k-1}, \rho_{k}\right)} \int_{\Omega \times \Omega} \frac{|x-y|^{p(x)}}{p(x) h^{p(x)-1}} \mathrm{~d} \gamma
$$

admits a unique solution $\gamma_{k}$ in $\Pi\left(\rho_{k-1}, \rho_{k}\right)$ and that $\operatorname{supp}_{k}$ satisfy

$$
\operatorname{supp}_{k} \subset\left\{(x, y) \in \Omega \times \Omega, \quad y=x-h\left|\nabla_{x}\left(G^{\prime}\left(\rho_{k}\right)+V\right)\right|^{q(x)-2} \nabla_{x}\left(G^{\prime}\left(\rho_{k}\right)+V\right)\right\}
$$

where $q(x)=(p(x) / p(x)-1)$.

Here, $V \in W^{1, \infty}(\Omega)$ being not necessarily smooth, we approximate $V$ by $C^{2}(\Omega)$-functions, and we use descent algorithm (6), the maximum principle $N \leq \rho_{k} \leq M$, and compact embedding $W^{1, q(x)}(\Omega) \hookrightarrow \hookrightarrow L^{q(x)}(\Omega)$ to establish $(10)$.
(3) We now use the maximum principle $N \leq \rho_{k} \leq M$ and (10) to prove that the sequence $\left(\rho_{k}\right)_{k}$ is a time discretization of the nonlinear $q(x)$-Laplacian parabolic equation, that is, for all test functions, $\phi \in C_{c}^{\infty}\left(\mathbb{R}^{d}\right)$,

$$
\int_{\Omega}\left(\rho_{k}-\rho_{k-1}\right) \phi \mathrm{d} x+h \int_{\Omega}<\left|\nabla_{x}\left(G^{\prime}\left(\rho_{k}\right)+V\right)\right|^{q(x)-2} \nabla_{x}\left(G^{\prime}\left(\rho_{k}\right)+V\right), \quad \nabla_{x} \phi>\rho_{k} \mathrm{~d} x=A_{k}^{h}(\phi),
$$


where $A_{k}^{h}(\phi)$ converges to 0 when $h$ tends to 0 .

(4) We define $\rho^{h}(t, x)$ by

$$
\left.\left.\rho^{h}(t, x)=\rho_{k}, \quad \text { if } t \in\right] h(k-1), h k\right], \rho(0, x)=\rho_{0} .
$$

Afterward, we establish the following:

(i) The strong convergence of the sequence $\left(\rho^{h}\right)_{h}$ to a function $\rho(t, x)$ in $L^{q(x)}([0, T] \times \Omega)$ for $0<T<\infty$

(ii) The weak convergence of nonlinear term $\left(\left.\operatorname{div}_{x}\left(\rho^{h} \mid\right) \nabla_{x}\left(G^{\prime}\left(\rho^{h}\right)+V\right)\right|^{q(x)-2} \nabla_{x}\left(G^{\prime}\left(\rho^{h}\right)+V\right)\right)_{h}$ to $\left.\operatorname{div}_{x}(\rho \mid) \nabla_{x}\left(G^{\prime}\left(\rho^{h}\right)+V\right)\right|^{q(x)-2} \nabla_{x}\left(G^{\prime} \rho+V\right)$ in $\left[C_{c}^{\infty}\left(\mathbb{R} \times \mathbb{R}^{d}\right)\right]^{\prime}$

To prove (i), we use descent algorithm (6) and the maximum principle $N \leq \rho^{h} \leq M$ to deduce that the sequence $\left(\rho^{h}\right)_{h}$ is bounded in $W^{1, q(x)}([0, T] \times \Omega)$ for $0<T<\infty$. Then, taking into account the compact embedding $W^{1, q(x)}([0, T] \times \Omega) \hookrightarrow \hookrightarrow L^{q(x)}([0, T] \times \Omega)$ established by Fan and Zhao in [9], we conclude that the sequence $\left(\rho^{h}\right)_{h}$ converges strongly to $\rho(t, x)$ in $L^{q(x)}([0, T] \times \Omega)$.

We now use (i) and the fact that $v \longmapsto|v|^{q(x)}$ is convex for all $x \in \Omega$ fixed to establish (ii). We combine (i) and (ii) to prove that the sequence $\left(\rho^{h}\right)_{h}$ converges to a weak solution of the $q(x)$-Laplacian parabolic equation.

Finally, we use the energy method to study the convergence of solutions of the $q(x)$-Laplacian parabolic equation to $\rho_{\infty}$ when $t \longrightarrow \infty$, where $\rho_{\infty} \in P(\Omega)$ is the probability density which satisfies $\rho_{\infty} \nabla_{x}\left(G^{\prime}\left(\rho_{\infty}\right)+V\right)=0$.

Note that in $[10,11]$, the authors proved a convergence of solutions to the equilibrium without specifying the speed of convergence. In [11], the long-time behavior of solutions of the $q(x)$-Laplacian parabolic equation is only established if $2 \leq q_{-} \leq q_{+}$.

In this paper, we extend to the variable exponent $q():. \Omega \longrightarrow] 1,+\infty\left[\right.$ such that $1<q_{-}<q_{+}<\infty$, the results obtained by the previous authors, and we also specify the rates of convergence.

Our results in this work are stated as follows:

Theorem 1. Assume that $\left(H_{\rho_{0}}\right),\left(H_{G}\right),\left(H_{q(.)}^{p(.)}\right)$, and $\left(H_{V}^{1}\right)$ hold, and define $\rho^{h}(t, x):[0,+\infty[\times \Omega \longrightarrow[0,+\infty[$ by

$$
\left.\left.\rho^{h}(t, x)=\rho_{k}(x), \quad \text { if } t \in\right] h(k-1) ; \mathrm{hk}\right], \rho(0, x)=\rho_{0}(x) .
$$

Then, the sequence $\left(\rho^{h}\right)_{h}$ converges to a weak solution $\rho(t, x)$ of the $q(x)$-Laplacian parabolic equation.

Theorem 2. Assume that $\left(H_{\rho_{0}}\right),\left(H_{G}\right),\left(H_{q(.)}^{p(.)}\right),\left(H_{V}^{1}\right)$, and $\left(H_{V}^{2}\right)$ hold. Let $\rho(t, x)$ be a weak solution of the $q(x)$-Laplacian parabolic equation. Define

$$
\begin{aligned}
& I_{1}:=\left\{t \in \left[0,+\infty\left[, \quad\left\|\left(\nabla_{x}\left(G^{\prime}(\rho(t, .))\right)+V\right)\right\|_{L_{\rho(t,)}^{q(.)}(\Omega)} \geq 1\right\},\right.\right. \\
& I_{2}:=\left\{t \in \left[0,+\infty\left[, \quad\left\|\left(\nabla_{x}\left(G^{\prime}(\rho(t, .))\right)+V\right)\right\|_{L_{\rho(t,)}^{q(.)}(\Omega)} \leq 1\right\} .\right.\right.
\end{aligned}
$$

Then,

$$
\begin{aligned}
& \left.\left[E\left(\rho\left(t_{2}, .\right)\right)-E\left(\rho_{\infty}\right)\right] \leq \frac{\left[E(\rho(t, .))-E\left(\rho_{\infty}\right)\right]}{\left[1+(\lambda / 2)^{p_{+} / q_{+}}\left(q_{+}-1\right)\left(E\left(\rho\left(t_{2}, .\right)\right)\right)-E\left(\rho_{\infty}\right)\left(q_{+}-1\right)\right.}\left(t-t_{2}\right)\right]^{\left(1 / q_{+}-1\right)}, \text { on } I_{2}, \\
& W_{p(.)}^{p_{+}}\left(\rho(t, .), \rho_{\infty}(.)\right) \leq \frac{2^{p_{+}}\left[E\left(\rho\left(t_{2}, .\right)\right)-E\left(\rho_{\infty}\right)\right]}{\lambda\left[1+(\lambda / 2)^{p_{+} / q_{+}}\left(q_{+}-1\right)\left(E\left(\rho\left(t_{2}, .\right)\right)\right)-E\left(\rho_{\infty}\right)\left(q_{+}^{-1)}\left(t-t_{2}\right)\right]^{\left(1 / q_{+}-1\right)}, \quad \text { on } I_{2},\right.} \\
& {\left[E(\rho(t, .))-E\left(\rho_{\infty}\right)\right] \leq e^{-(\lambda / 2)^{p_{+}^{\prime}}\left(t-t_{1}\right)}\left[E\left(\rho_{0}\right)-E\left(\rho_{\infty}\right)\right], \quad \text { on } I_{1},} \\
& W_{p(.)}^{p_{+}}\left(\rho(t, .), \rho_{\infty}(.)\right) \leq \frac{2^{p_{+}}}{\lambda} e^{-(\lambda / 2)^{p_{+}^{\prime}}\left(t-t_{1}\right)}\left[E\left(\rho_{0}\right)-E\left(\rho_{\infty}\right)\right], \quad \text { on } I_{1},
\end{aligned}
$$

where $t_{1}:=\inf I_{1}$ and $t_{2}:=\inf I_{2}$

\section{Preliminaries}

2.1. Assumptions. Throughout this work, we will assume the following:

$\left(H_{\rho_{0}}\right) \rho_{0}$ is a probability density on $\Omega$, with $N \leq \rho_{0} \leq M$, for some $0<N<M$, and $\int_{\Omega} G\left(\rho_{0}\right) \mathrm{d} x<\infty$.

$\left.\left(H_{q(.)}^{p(.)}\right): q(),. p():. \Omega \longrightarrow\right] 1,+\infty[$ are continuous functions such that $((1 / p(x))+(1 / q(x)))=1$ for all $x \in \Omega, \quad$ and $\quad 1<p:=\min _{\Omega} p(x) \leq \max _{\Omega} p(x):=p_{+}$; $1<q:=\min _{\Omega} q(x) \leq \max _{\Omega} q(x):=q_{+}$.

$\left(H_{G}\right) \quad G:[0,+\infty[\longrightarrow \mathbb{R}$ is strictly convex, $G \in C^{2}(] 0,+\infty[)$, and $t \longmapsto t^{d} G\left(t^{-d}\right)$ is convex.

$$
\left(H_{V}^{1}\right) V \in W^{1, \infty}(\Omega) .
$$

$\left(H_{V}^{2}\right) \quad V: \mathbb{R}^{d} \longrightarrow \mathbb{R}$ is a potential which satisfies $V(0)=0, V \in C^{2}(\Omega)$, and 


$$
\begin{array}{r}
V(x)-V(y) \geq \nabla V(y) .(x-y)+\lambda|x-y|^{p_{+}}, \\
\text {for all } x, y \in \Omega, \text { for some } \lambda>0 .
\end{array}
$$

2.2. Lebesgue-Sobolev Spaces with Variable Exponents. We recall in this section some definitions and fundamental properties of the Lebesgue and Sobolev space with variable exponents.

Definition 1 . Let $\rho$ be a probability measure on $\Omega$ and $p():. \Omega \longrightarrow] 1,+\infty[$ be a measurable function. We denote by $L_{\rho}^{p(.)}(\Omega)$ the Lebesgue space with the variable exponent defined by

$$
L_{\rho}^{p(.)}(\Omega):=\left\{u: \Omega \longrightarrow \mathbb{R} ; \int_{\Omega}\left|\frac{u(x)}{\lambda}\right|^{p(x)} \rho(x) \mathrm{d} x \leq 1,\right.
$$

$$
\text { for some } \lambda>0\} \text {, }
$$

with the norm

$$
\|u\|_{L_{\rho}^{p(.)}(\Omega)}=\inf \left\{\lambda>0, \int_{\Omega}\left|\frac{u(x)}{\lambda}\right|^{p(x)} \rho(x) \mathrm{d} x \leq 1\right\},
$$

for all $u \in L_{\rho}^{p(.)}(\Omega)$.

We denote by $W_{\rho}^{1, p(.)}(\Omega)$ the Sobolev space with the variable exponent defined by

$$
W_{\rho}^{1, p(.)}(\Omega):=\left\{u \in L_{\rho}^{p(.)}(\Omega), \quad|\nabla u| \in L_{\rho}^{p(.)}(\Omega)\right\},
$$

equipped with the norm

$$
\|u\|_{W_{\rho}^{1, p(.)}(\Omega)}:=\|u\|_{L_{\rho}^{p(.)}(\Omega)}+\|\nabla u\|_{L_{\rho}^{p(.)}(\Omega)} .
$$

In particular, if $\rho(x)=1$, then $L_{\rho}^{p(.)}(\Omega)=L^{p(.)}(\Omega)$ and $W_{\rho}^{1, p(.)}(\Omega)=W^{1, p(.)}(\Omega)$.

We also define the space $W_{0}^{1, p(.)}(\Omega)$ as the closure of the space of $C^{\infty}$-functions with compact support in $\Omega C_{c}^{\infty}(\Omega)$ in the space $W^{1, p(.)}(\Omega)$ endowed with the norm

$$
\|u\|_{W_{0}^{1, p(.)}(\Omega)}:=\|\nabla u\|_{W^{1, p(.)}(\Omega)} .
$$

It is well known (cf. [9]) that $L_{\rho}^{p(.)}(\Omega)$ and $W_{\rho}^{1, p(.)}(\Omega)$ are Banach spaces, respectively, with norms (20) and (22).

Proposition 1 (Hölder inequality; see [12]). Let $\rho \in P(\Omega)$ and $p(),. q():. \Omega \longrightarrow] 1,+\infty[$ be two measurable functions such that $((1 / p(x))+(1 / q(x)))=1$, for all $x \in \Omega$.

If $u \in L_{\rho}^{p(.)}(\Omega)$ and $v \in L_{\rho}^{q(.)}(\Omega)$, then

$$
\int_{\Omega}|u(x) v(x)| \rho(x) \mathrm{d} x \leq\left(\frac{1}{p_{-}}+\frac{1}{q_{-}}\right)\|u\|_{L_{\rho}^{p(.)}(\Omega)}\|v\|_{L_{\rho}^{q(.)}(\Omega)} .
$$

Furthermore, if $\left.p_{1}(),. p_{2}(),. p_{3}():. \Omega \longrightarrow\right] 1,+\infty[$ are measurable functions such that $\left(1 / p_{1}(x)\right)=\left(1 / p_{2}(x)\right)+\left(1 / p_{3}(x)\right)$, for almost all $x \in \Omega$, we have

$$
\|u v\|_{L_{\rho}^{p_{1}(.)}(\Omega)} \leq 2\|u\|_{L_{\rho}^{p_{2}(.)}(\Omega)}\|v\|_{L_{\rho}^{p_{3}(.)}(\Omega)},
$$

for $u \in L_{\rho}^{p_{2}(.)}(\Omega)$ and $v \in L_{\rho}^{p_{3}(.)}(\Omega)$.

Proposition 2 (see [9]). Let $\rho \in P(\Omega)$ and $p_{1}(),. p_{2}():. \Omega \longrightarrow[1,+\infty[$ be two measurable functions such that $p_{1}(x) \leq p_{2}(x)$ on $\Omega$. Then, we have the following continuous injection:

$$
L_{\rho}^{p_{2}(.)}(\Omega) \longmapsto L_{\rho}^{p_{1}(.)}(\Omega) .
$$

Furthermore,

$$
\|u\|_{L_{\rho}^{p_{1}(.)}(\Omega)} \leq 2\|u\|_{L_{\rho}^{p_{2}(.)}(\Omega)},
$$

for all $u \in L_{\rho}^{p_{2}(.)}(\Omega)$.

Proposition 3 (see [9]). Assume that $\left(H_{q(.)}^{p(.)}\right)$ holds. Then, the following statements hold:

(i) The Banach spaces $W_{\rho}^{1, p(x)}(\Omega)$ and $L_{\rho}^{p(.)}(\Omega)$ are separable and reflexive Banach spaces

(ii) The embedding $W^{1, q(x)}(\Omega) \hookrightarrow \hookrightarrow L^{q(x)}(\Omega)$ is continuous and compact

(iii) There is a constant $C(\Omega)$ such that $\|u\|_{W^{1, q(x)}(\Omega)} \leq$ $C(\Omega)\|\nabla u\|_{L^{q(x)}(\Omega)}$ for all $u \in W_{0}^{1, q(x)}(\Omega)$

\section{Existence of Solutions for the Nonlinear $q(x)$ - Laplacian Parabolic Equation}

In this section, we prove the existence of solutions for the nonlinear $q(x)$-Laplacian parabolic equation.

3.1. Euler-Lagrangian Equation for Variational Problem (37). Here, we show that the sequence $\left(\rho_{k}\right)_{k}$ defined in (37) is a time discretization of the $q(x)$-Laplacian parabolic equation, i.e., for all test functions, $\phi \in C_{c}^{\infty}\left(\mathbb{R}^{d}, \mathbb{R}\right)$, we have

$$
\begin{gathered}
\int_{\Omega}\left(\rho_{k}-\rho_{k-1}\right) \phi \mathrm{d} x+h \int_{\Omega}<\left|\nabla_{x}\left(G^{\prime}\left(\rho_{k}\right)+V\right)\right|^{q(x)-2} \\
\cdot \nabla_{x}\left(G^{\prime}\left(\rho_{k}\right)+V\right), \quad \nabla_{x} \phi>\rho_{k} \mathrm{~d} x=A_{k}^{h}(\phi),
\end{gathered}
$$

where $A_{k}^{h}(\phi)$ converges to 0 when $h$ tends to 0 .

Lemma 1. Assume that $\left(H_{\rho_{0}}\right),\left(H_{G}\right)$, and $\left(H_{V}^{1}\right)$ hold. Then, the variational problem

$$
\left(P_{1}\right): \inf _{\rho \in P(\Omega)}\left\{I(\rho):=\int_{\Omega}(G(\rho)+\rho V) \mathrm{d} x+K_{p(.)}^{h}\left(\rho_{0}, \rho\right)\right\},
$$

where

$$
K_{p(.)}^{h}\left(\rho_{0}, \rho\right):=\inf _{\gamma \in \Pi\left(\rho_{0}, \rho\right)} \int_{\Omega \times \Omega} \frac{|x-y|^{p(x)}}{p(x) h^{p(x)-1}} \mathrm{~d} \gamma,
$$

admits a unique solution $\rho_{1}$ in $P(\Omega)$ which satisfies $N \leq \rho_{1} \leq M$. 
Proof 1. Since $N \leq \rho_{0} \leq M$, then the solution $\rho_{1}$ of variational problem $\left(P_{1}\right)$ (if there exists) satisfies $N \leq \rho_{1} \leq M$. The proof of the maximum principle is carried out similarly as given in [7].

Since $\int_{\Omega} G\left(\rho_{0}\right) \mathrm{d} x<\infty$ and $V \in L^{\infty}(\Omega)$, we have $I\left(\rho_{0}\right)<\infty$.

Let $\rho \in P(\Omega)$; since $G$ is convex and $V \in L^{\infty}(\Omega)$,

$$
I(\rho) \geq \int_{\Omega}[G(\rho)+\rho V] \mathrm{d} x \geq|\Omega| G\left(\frac{1}{|\Omega|}\right)-\|V\|_{L^{\infty}(\Omega)} .
$$

We conclude that inf $\operatorname{in}_{\rho \in P(\Omega)} I(\rho)$ is finite.

Let $\left(\rho^{n}\right)_{n}$ be a sequence in $P(\Omega)$ such that $N \leq \rho^{n} \leq M$ and $I\left(\rho^{n}\right)$ converges to inf $\operatorname{s\in P(\Omega )} I(\rho)$. Then, $\left(\rho^{n}\right)_{n}$ converges weakly* to $\rho_{1}$ in $L^{\infty}(\Omega)$ up to a subsequence.

$G$ being positive and convex, then

$$
\begin{aligned}
& \int_{\Omega}\left[G\left(\rho^{n}\right)+\rho^{n} V\right] \mathrm{d} x \geq \int_{\Omega}\left[G\left(\rho_{1}\right)+\rho_{1} V\right] \mathrm{d} x \\
& +\int_{\Omega} G^{\prime}\left(\rho_{1}\right)\left(\rho^{n}-\rho_{1}\right) \mathrm{d} x+\int_{\Omega}\left(\rho^{n}-\rho_{1}\right) V \mathrm{~d} x .
\end{aligned}
$$

Next, we use the fact that $G^{\prime}\left(\rho_{1}\right) \in L^{\infty}(\Omega)$ and $V \in L^{\infty}(\Omega)$ to obtain

$$
\liminf _{n \rightarrow \infty} \int_{\Omega}\left[G\left(\rho^{n}\right)+\rho^{n} V\right] \mathrm{d} x \geq \int_{\Omega}\left[G\left(\rho_{1}\right)+\rho_{1} V\right] \mathrm{d} x .
$$

Since the variable exponent $p($.$) is continuous on \Omega$, the Kantorovich problem

$$
(K): \inf _{\gamma \in \Pi\left(\rho_{0}, \rho^{n}\right)} \int_{\Omega \times \Omega} \frac{|x-y|^{p(x)}}{p(x) h^{p(x)-1}} \mathrm{~d} \gamma,
$$

admits a solution $\gamma^{n}$. Moreover, since $\Omega$ is bounded, the sequence $\left(\gamma^{n}\right)_{n}$ converges to a measure $\gamma_{1}$ in $P(\Omega \times \Omega)$ narrowly, and $\gamma_{1} \in \Pi\left(\rho_{0}, \rho_{1}\right)$; and then, we derive that

$$
\liminf _{n \longrightarrow \infty} K_{p(.)}^{h}\left(\rho_{0}, \rho^{n}\right) \geq \int_{\Omega \times \Omega} \frac{|x-y|^{p(x)}}{p(x) h^{p(x)-1}} \mathrm{~d} \gamma_{1} \geq K_{p(.)}^{h}\left(\rho_{0}, \rho_{1}\right) \text {. }
$$

We combine (35) and (33) to obtain

$$
\liminf _{n \longrightarrow \infty} I\left(\rho^{n}\right) \geq I\left(\rho_{1}\right) .
$$

Thus, $\rho_{1}$ is a solution of variational problem $\left(P_{1}\right)$. From the strict convexity of $G$, we deduce that $\rho \longmapsto \int_{\Omega}(G(\rho)+$ $\rho V) \mathrm{d} x$ is strictly convex and so is $\rho \longmapsto I(\rho)$ on $P(\Omega)$, and consequently, the uniqueness of the solution $\rho_{1}$ of $\left(P_{1}\right)$ follows.

Now, we assume that $\left(H_{\rho_{0}}\right),\left(H_{G}\right)$, and $\left(H_{V}^{1}\right)$ hold. Then, from Lemma 1, we obtain that the variational problem

$$
\left(P_{k}\right): \inf _{\rho \in P(\Omega)}\left\{I(\rho):=\int_{\Omega}[G(\rho)+\rho V] \mathrm{d} x+K_{p(.)}^{h}\left(\rho_{k-1}, \rho\right)\right\},
$$

with

$$
K_{p(.)}^{h}\left(\rho_{k-1}, \rho\right):=\inf _{\gamma \in \Pi\left(\rho_{k-1}, \rho\right)} \int_{\Omega \times \Omega} \frac{|x-y|^{p(x)}}{p(x) h^{p(x)-1}} \mathrm{~d} \gamma,
$$

admits a unique solution $\rho_{k}$ for all $k \geq 1$.

Next, we prove that $\left(\rho_{k}\right)_{k}$ is a time discretization of the nonlinear $q(x)$-Laplacian parabolic equation. In order to achieve this, we use the following lemma.

Lemma 2. Assume that $\left(H_{\rho_{0}}\right),\left(H_{G}\right)$, and $\left(H_{V}^{1}\right)$ hold. Then, the Kantorovich problem

$$
(K): \inf _{\gamma \in \Pi\left(\rho_{k-1}, \rho_{k}\right)}\left\{\int_{\Omega \times \Omega} \frac{|x-y|^{p(x)}}{p(x) h^{p(x)-1}} \mathrm{~d} \gamma\right\},
$$

admits a solution $\gamma_{k}$ such that

$$
\operatorname{supp}_{k} \subset\left\{(x, y) \in \Omega \times \Omega, \quad y=x-h\left|\nabla_{x}\left(G^{\prime}\left(\rho_{k}\right)+V\right)\right|^{q(x)-2} \nabla_{x}\left(G^{\prime}\left(\rho_{k}\right)+V\right)\right\}
$$

where $q(x)=(p(x) / p(x)-1)$.

$$
\int_{\Omega \times \Omega} \phi(x, y) \mathrm{d} \gamma^{\epsilon}=\int_{\Omega \times \Omega} \phi\left(x, T_{\epsilon}(y)\right) \mathrm{d} \gamma_{k},
$$

Proof 2. The proof of Lemma 2 is derived following the two steps.

Step 1. We first assume that $V \in C^{2}(\Omega)$. by

Fix $\psi \in C_{c}^{\infty}\left(\mathbb{R}^{d}, \mathbb{R}^{d}\right)$. Let $\left(T_{\epsilon}\right)_{\epsilon \in \mathbb{R}}$ in $C^{\infty}\left(\mathbb{R}^{d}, \mathbb{R}^{d}\right)$ defined

$$
T_{\epsilon}(x)=x+\epsilon \psi(x)
$$

Define the probability density $\rho_{\epsilon}$ as $\rho_{\epsilon}=T_{\epsilon} \# \rho_{k}$ and $\gamma^{\epsilon}$ as a probability measure on $\Omega \times \Omega$ defined by for all $\phi \in C_{b}^{0}(\Omega \times \Omega)$.

For $\epsilon \neq 0$,

$$
\begin{aligned}
\frac{I\left(\rho_{\epsilon}\right)-I\left(\rho_{k}\right)}{\epsilon}= & \int_{\Omega} \frac{G\left(\rho_{\epsilon}\right)-G\left(\rho_{k}\right)}{\epsilon} \mathrm{d} x+\int_{\Omega} \frac{V\left(T_{\epsilon}\right)-V(x)}{\epsilon} \mathrm{d} x \\
& +\frac{1}{\epsilon}\left[K_{p(.)}^{h}\left(\rho_{\epsilon}, \rho_{k-1}\right)-K_{p(.)}^{h}\left(\rho_{k-1}, \rho_{k}\right)\right] .
\end{aligned}
$$

Since $G$ satisfies $\left(H_{G}\right)$, then 
$\lim _{\epsilon \longrightarrow 0} \int_{\Omega} \frac{G\left(\rho_{\epsilon}\right)-G\left(\rho_{k}\right)}{\epsilon} \mathrm{d} x=\int_{\Omega} \nabla_{x} G^{\prime}\left(\rho_{k}\right) \cdot \psi \rho_{k} \mathrm{~d} x$. [7])

Since $V \in C^{2}(\Omega)$, from the Taylor formula, we have (see

$$
\begin{aligned}
V\left(T_{\epsilon}(x)\right)-V(x)= & \epsilon \nabla_{x} V(x) \cdot \psi(x) \\
& +\epsilon^{2}(\psi(x))^{\tau} \nabla_{x}^{2} V(x+\epsilon \theta \psi(x)) \\
& \cdot \psi(x), \quad \text { with } \theta \in] 0,1[.
\end{aligned}
$$

Then, we have after integration,

$$
\begin{aligned}
\int_{\Omega} \frac{V\left(T_{\epsilon}\right)-V(x)}{\epsilon} \mathrm{d} x= & \int_{\Omega}<\nabla V(x), \psi>\rho_{k} \mathrm{~d} x \\
& +\epsilon \int_{\Omega}(\psi(x))^{\tau} \cdot \nabla^{2} V \\
& \cdot(x+\epsilon \theta \psi(x)) \cdot \psi(x) \rho_{k} \mathrm{~d} x .
\end{aligned}
$$

By using (41) and the fact that $V \in W^{1, \infty}(\Omega)$, we have $\left|(\psi(x))^{\tau} . \nabla^{2} V(x+\epsilon \theta \psi(x)) \cdot \psi(x)\right| \leq\left\|\nabla^{2} V\right\|_{L^{\infty}(\Omega)}\|\psi\|_{L^{\infty}(\Omega)}$. have

We now use the dominated convergence theorem to

$$
\lim _{\epsilon \longrightarrow 0} \int_{\Omega} \frac{V\left(T_{\epsilon}\right)-V(x)}{\epsilon} \mathrm{d} x=\int_{\Omega} \nabla V \cdot \psi \rho_{k} \mathrm{~d} x .
$$

Note that $\gamma^{\epsilon}$ defined in (42) belongs to $\Pi\left(\rho_{k-1}, \rho_{\epsilon}\right)$, and

$$
\begin{aligned}
K_{p(.)}^{h}\left(\rho_{k-1}, \rho_{k}\right) & =\int_{\Omega \times \Omega} \frac{|x-y|^{p(x)}}{p(x) h^{p(x)-1}} \mathrm{~d} \gamma_{k}, K_{p(.)}^{h}\left(\rho_{k-1}, \rho_{\epsilon}\right) \\
& \leq \int_{\Omega \times \Omega} \frac{\left|x-T_{\epsilon}(y)\right|^{p(x)}}{p(x) h^{p(x)-1}} \mathrm{~d} \gamma_{k} .
\end{aligned}
$$

So, for $\epsilon>0$, we have

$$
\begin{aligned}
& \frac{1}{\epsilon}\left[K_{p(.)}^{h}\left(\rho_{k-1}, \rho_{\epsilon}\right)-K_{p(.)}^{h}\left(\rho_{k-1}, \rho_{k}\right)\right] \\
& \quad \leq \frac{1}{\epsilon} \int_{\Omega \times \Omega}\left[\frac{\left|x-T_{\epsilon}(y)\right|^{p(x)}-|x-y|^{p(x)}}{p(x) h^{p(x)-1}}\right] \mathrm{d} \gamma_{k} .
\end{aligned}
$$

Note that

$$
\begin{aligned}
\lim _{\epsilon \longrightarrow 0^{+}} \frac{1}{\epsilon} \int_{\Omega \times \Omega}\left[\frac{\left|x-T_{\epsilon}(y)\right|^{p(x)}-|x-y|^{p(x)}}{p(x) h^{p(x)-1}}\right] d \gamma_{k} \\
\quad=-\int_{\Omega \times \Omega}<\left|\frac{x-y}{h}\right|^{p(x)-2}\left(\frac{x-y}{h}\right), \psi>d \gamma_{k} .
\end{aligned}
$$

Indeed,

(i) We have

$$
\begin{gathered}
\lim _{\epsilon \longrightarrow 0^{+}} \frac{1}{\epsilon}\left[\frac{|| x-\left.T_{\epsilon}(y)\right|^{p(x)}-|x-y|^{p(x)}}{p(x) h^{p(x)-1}}\right] \\
=-\left|\frac{x-y}{h}\right|^{p(x)-2}\left(\frac{x-y}{h}\right) \cdot \psi .
\end{gathered}
$$

(ii) On the contrary, the Taylor formula with respect to $\epsilon$ enables us to write

$$
\begin{aligned}
\frac{|| x-\left.T_{\epsilon}(y)\right|^{p(x)}-|x-y|^{p(x)}}{p(x) h^{p(x)-1}}= & -\epsilon\left|\frac{x-y-\theta \epsilon \psi(y)}{h}\right|^{p(x)-2} \\
& \cdot\left(\frac{x-y-\theta \epsilon \psi(y)}{h}\right) \cdot \psi(y),
\end{aligned}
$$

where $\theta \epsilon(0,1)$ and $T_{\epsilon}(y)=y+\epsilon \psi(y)$. Also, we have

$$
\left|\frac{\left|x-T_{\epsilon}(y)\right|^{p(x)}-|x-y|^{p(x)}}{\epsilon p(x) h^{p(x)-1}}\right|
$$

$$
\begin{aligned}
& =\left|\frac{x-y-\theta \epsilon \psi(y)}{h}\right|^{p(x)-1}|\psi(y)| \\
& \leq\left[\left|\frac{x-y-\theta \epsilon \psi(y)}{h}\right|+1\right]^{p(x)-1}|\psi(y)| \\
& \leq\left[\left|\frac{x-y-\theta \epsilon \psi(y)}{h}\right|+1\right]^{p(x)}|\psi(y)| \\
& \leq 2^{p_{+}-1}\left|\frac{x-y}{h}\right|^{p(x)}+2^{p_{+}-1}\left(\frac{|\psi(y)|}{h}+1\right)^{p_{+}}|\psi(y)|,
\end{aligned}
$$

with

$$
\int_{\Omega \times \Omega}\left[2^{p_{+}-1}\left|\frac{x-y}{h}\right|^{p(x)}+2^{p_{+}-1}\left(\frac{|\psi(y)|}{h}+1\right)^{p_{+}}|\psi(y)|\right] \mathrm{d} \gamma_{k}
$$

We then combine the results given in (i) and (ii) and the dominated convergence theorem to obtain (50).

Since $\rho_{k}$ minimizes $I$ on $P(\Omega)$, then

$$
\lim _{\epsilon \longrightarrow 0} \frac{I\left(\rho_{\epsilon}\right)-I\left(\rho_{k}\right)}{\epsilon}=0 .
$$

So, we combine (44), (48), and (50) to obtain that

$$
\begin{gathered}
0=\lim _{\epsilon \longrightarrow 0^{+}} \frac{I\left(\rho_{\epsilon}\right)-I\left(\rho_{k}\right)}{\epsilon} \leq \int_{\Omega} \nabla_{x}\left(G^{\prime}\left(\rho_{k}\right)+V\right) \cdot \psi \rho_{k} \mathrm{~d} x \\
-\int_{\Omega \times \Omega}<\left|\frac{x-y}{h}\right|^{p(x)-2}\left(\frac{x-y}{h}\right), \\
\psi>\mathrm{d} \gamma_{k} .
\end{gathered}
$$


Replacing $\psi$ by $-\psi$ in (56), we get

$$
\begin{aligned}
\int_{\Omega} & <\nabla_{x}\left(G^{\prime}\left(\rho_{k}\right)+V\right), \psi>\rho_{k} d x \\
& -\int_{\Omega \times \Omega}<\left|\frac{x-y}{h}\right|^{p(x)-2}\left(\frac{x-y}{h}\right), \quad \psi>\mathrm{d} \gamma_{k} \leq 0 .
\end{aligned}
$$

From (56) and (57), we deduce that

$$
\begin{aligned}
\int_{\Omega} & <\nabla_{x}\left(G^{\prime}\left(\rho_{k}\right)+V\right), \psi>\rho_{k} \mathrm{~d} x \\
& +\int_{\Omega \times \Omega}<\left|\frac{y-x}{h}\right|^{p(x)-2}\left(\frac{y-x}{h}\right), \quad \psi>\mathrm{d} \gamma_{k}=0 .
\end{aligned}
$$

Thus, we obtain (40) when $V \in C^{2}(\Omega)$.

Now, let us establish the proof of the lemma when $V \in W^{1, \infty}(\Omega)$ and is nonregular.
Step 2. Assume that $V \in W^{1, \infty}(\Omega)$. Let $\left(V^{\delta}\right)_{\delta}$ be a sequence in $C^{2}(\Omega)$ such that $\lim _{\delta \rightarrow 0}\left\|V^{\delta}-V\right\|_{W^{1, \infty}(\Omega)}=0$.

By fixing $\delta>0$, we define the sequence $\left(\rho_{k}^{\delta}\right)_{k}$ such that $\rho_{0}^{\delta}=\rho_{0}$ with $\rho_{k}^{\delta}$ (for $k \geq 1$ ), the solution of the variational problem

$$
\left(P_{k}^{\delta}\right): \inf _{\rho \in P(\Omega)}\left[\int_{\Omega}\left(G(\rho)+\rho V^{\delta}\right) \mathrm{d} x+K_{p(.)}^{h}\left(\rho_{k-1}^{\delta}, \rho\right)\right],
$$

where $K_{p(.)}^{h}\left(\rho_{k-1}^{\delta}, \rho\right)$ is defined as in (38). As in Lemma 1, the variational problem $\left(P_{k}^{\delta}\right)$ admits a unique solution $\rho_{k}^{\delta}$ in $P(\Omega)$, and $N \leq \rho_{k}^{\delta} \leq M$. Hence, the Kantorovich problem

$$
K^{\delta}: \inf _{\gamma \in \Pi\left(\rho_{k-1}^{\delta}, \rho_{k}^{\delta}\right)}\left[\int_{\Omega \times \Omega} \frac{|x-y|^{p(x)}}{p(x) h^{p(x)-1}} \mathrm{~d} \gamma\right]
$$

admits a solution $\gamma_{k}^{\delta}$ such that

$$
\operatorname{supp}_{k}^{\delta} \subset\left\{(x, y) \in \Omega \times \Omega, y=x-h\left|\nabla_{x}\left(G^{\prime}\left(\rho_{k}^{\delta}\right)+V^{\delta}\right)\right|^{q(x)-2} \nabla_{x}\left(G^{\prime}\left(\rho_{k}^{\delta}\right)+V^{\delta}\right)\right\}
$$

Let us show that $\left(\rho_{k}^{\delta}\right)_{\delta}$ converges to $\rho_{k}$ and $\left(\gamma_{k}^{\delta}\right)_{\delta}$ conUsing $\left(P_{k}^{\delta}\right)$ and (61), we have verges to $\gamma_{k}$ up to a subsequence, as well as $\operatorname{supp} \gamma_{k}$ satisfies (40)

$$
\int_{\Omega}\left(G\left(\rho_{k-1}^{\delta}\right)+\rho_{k-1}^{\delta} V^{\delta}\right) \mathrm{d} x-\int_{\Omega}\left(G\left(\rho_{k}^{\delta}\right)+\rho_{k}^{\delta} V^{\delta}\right) \mathrm{d} x \geq h \int_{\Omega} \frac{\left|\nabla_{x}\left(G^{\prime}\left(\rho_{k}^{\delta}\right)+V^{\delta}\right)\right|^{q(x)}}{p(x)} \rho_{k}^{\delta} \mathrm{d} x
$$

However, $G$ is convex, and $V \in W^{1, \infty}(\Omega)$; and then,

$$
\int_{\Omega}\left|\nabla_{x}\left(G^{\prime}\left(\rho_{k}^{\delta}\right)+V^{\delta}\right)\right|^{q(x)} \rho_{k}^{\delta} \leq \frac{p_{+}}{h}\left[\int_{\Omega} G\left(\rho_{0}\right) \mathrm{d} x-|\Omega| G\left(\frac{1}{|\Omega|}\right)+2\|V\|_{L^{\infty}(\Omega)}\right] .
$$

Furthermore, recalling (63) and the fact that $N \leq \rho_{k}^{\delta} \leq M$, $\nabla_{x}\left(G^{\prime}\left(\rho_{k}^{\delta}\right)\right)=G^{\prime \prime}\left(\rho_{k}^{\delta}\right) \nabla_{x}\left(\rho_{k}^{\delta}\right)$, and $\quad\left|\nabla_{x}\left(G^{\prime}\left(\rho_{k}^{\delta}\right)\right)\right|^{q(x)} \leq$ $2^{q_{+}-1}\left[\left|\nabla_{x}\left(G^{\prime}\left(\rho_{k}^{\delta}\right)+V^{\delta}\right)\right|^{q^{q(x)}}+\left|\nabla V^{\delta}\right|^{q(x)}\right]$, we obtain

$$
\int_{\Omega}\left|\nabla_{x} \rho_{k}^{\delta}\right|^{q(x)} \mathrm{d} x \leq \frac{2^{q_{+}-1}}{k_{0}}\left[\frac{p_{+}}{\operatorname{Nh}}\left(\int_{\Omega} G\left(\rho_{0}\right) \mathrm{d} x-|\Omega| G\left(\frac{1}{|\Omega|}\right)+2\|V\|_{L^{\infty}(\Omega)}\right)+\|\nabla V\|_{L^{\infty}(\Omega)}^{q_{+}}\right],
$$

where $k_{0}=\inf _{(t, x) \in[N, M] \times \Omega}\left[G^{\prime \prime}(t)\right]^{q(x)}$. We conclude that $\left(\rho_{k}^{\delta}\right)_{\delta}$ is bounded on $W^{1, q(x)}(\Omega)$. Since $q($.$) is continuous$ and $\Omega$ is a bounded set, the injection $W^{1, q(x)}(\Omega) \hookrightarrow \hookrightarrow L^{q(x)}(\Omega)$ is compact (see [9]), and hence, the sequence $\left(\rho_{k}^{\delta}\right)_{\delta}$ converges strongly to some $u_{k}$ in $L^{q(x)}(\Omega)$ up to a subsequence. Moreover, $\rho_{k}^{\delta}$ minimizes $\left(P_{k}^{\delta}\right)$ for $k \in \mathbb{N}^{*}$, and then, we have

$$
\begin{aligned}
& \int_{\Omega}\left(G(\rho)+\rho V^{\delta}\right) \mathrm{d} x+K_{p(.)}^{h}\left(\rho_{k-1}^{\delta}, \rho\right) \\
& \geq \int_{\Omega}\left(G\left(\rho_{k}^{\delta}\right)+\rho_{k}^{\delta} V^{\delta}\right) \mathrm{d} x+K_{p(.)}^{h}\left(\rho_{k-1}^{\delta}, \rho_{k}^{\delta}\right),
\end{aligned}
$$

for all $\rho \in P(\Omega)$. Also, from the boundedness of $\Omega,\left(\gamma_{k}^{\delta}\right)_{\delta}$ converges narrowly to a measure $\Gamma_{k}$ in $P(\Omega \times \Omega)$, and 
$\Gamma_{k} \in \Pi\left(u_{k-1}, u_{k}\right)$. Then, we use the strong convergence of $\left(\rho_{k}^{\delta}\right)_{\delta}$ to $u_{k}$ and the fact that $\left\|V^{\delta}-V\right\|_{W^{1, \infty}(\Omega)}$ converges to 0 when $\delta \longrightarrow 0$ to obtain that

$$
\begin{aligned}
& \int_{\Omega}(G(\rho)+\rho V) \mathrm{d} x+K_{p(.)}^{h}\left(u_{k-1}, \rho\right) \\
& \quad \geq \int_{\Omega}\left(G\left(u_{k}\right)+u_{k} V\right) \mathrm{d} x+K_{p(.)}^{h}\left(u_{k-1}, u_{k}\right),
\end{aligned}
$$

for all $\rho \in P(\Omega)$. Since $\rho_{0}^{\delta}=\rho_{0}$, we conclude that $u_{k}=\rho_{k}$ and $\Gamma_{k}=\gamma_{k}$ for all $k \in \mathbb{N}^{*}$.

By using (63) and $N \leq \rho_{k} \leq M$, we deduce that $\left(\nabla_{x}\left(G^{\prime}\left(\rho_{k}^{\delta}\right)+V^{\delta}\right)\right)_{\delta}$ is bounded in $\left[L^{q(x)}(\Omega)\right]^{d}$. Since $\left(\rho_{k}^{\delta}\right)_{\delta}$ converges strongly to $\rho_{k}$ in $L^{q(x)}(\Omega)$ and $G^{\prime}$ is continuous, then $\left(\nabla_{x}\left(G^{\prime}\left(\rho_{k}^{\delta}\right)+V^{\delta}\right)\right)_{\delta}$ converges weakly to $\nabla_{x}\left(G^{\prime}\left(\rho_{k}\right)+\right.$ $V)$ in $\left[L^{q(x)}(\Omega)\right]^{d}$.

Next, we use (57), and we have

$$
\begin{aligned}
\int_{\Omega} & <\nabla_{x}\left(G^{\prime}\left(\rho_{k}^{\delta}\right)+V^{\delta}\right), \psi>\rho_{k}^{\delta} \mathrm{d} x \\
& +\int_{\Omega \times \Omega}<\left|\frac{y-x}{h}\right|^{p(x)-2}\left(\frac{y-x}{h}\right), \quad \psi>d \gamma_{k}^{\delta}=0 .
\end{aligned}
$$

Since $\left(\rho_{k}^{\delta}\right)_{\delta}$ converges strongly to $\rho_{k}$ in $L^{q(x)}(\Omega)$, $\left(\nabla_{x}\left(G^{\prime}\left(\rho_{k}^{\delta}\right)+V^{\delta}\right)\right)_{\delta}$ converges weakly to $\nabla_{x}\left(G^{\prime}\left(\rho_{k}\right)+V\right)$ in
$\left[L^{q(x)}(\Omega)\right]^{d}$, and $\left(\gamma_{k}^{\delta}\right)_{\delta}$ converges narrowly to $\gamma_{k}$ in $P(\Omega \times \Omega)$; then, tending $\delta$ to 0 in (67), we obtain

$$
\begin{aligned}
\int_{\Omega} & <\nabla_{x}\left(G^{\prime}\left(\rho_{k}\right)+V^{\delta}\right), \psi>\rho_{k} \mathrm{~d} x \\
& +\int_{\Omega \times \Omega}<\left|\frac{y-x}{h}\right|^{(x)-2}\left(\frac{y-x}{h}\right), \quad \psi>d \gamma_{k}=0,
\end{aligned}
$$

for all test functions, $\psi \in C_{c}^{\infty}\left(\mathbb{R}^{d}, \mathbb{R}^{d}\right)$.

Finally, we obtain the equality

$$
y=x-h\left|\nabla_{x}\left(G^{\prime}\left(\rho_{k}\right)+V\right)\right|^{q(x)-2} \nabla_{x}\left(G^{\prime}\left(\rho_{k}\right)+V\right) \gamma_{k} \text { a.e. }
$$

Now, let us prove that $\left(\rho_{k}\right)_{k}$ is a time discretization of a nonlinear $q(x)$-Laplacian parabolic equation. Let $\psi \in C_{c}^{\infty}\left(\mathbb{R}^{d}, \mathbb{R}\right)$ be a test function; we have

$$
\int_{\Omega}\left(\rho_{k}-\rho_{k-1}\right) \psi \mathrm{d} x=\int_{\Omega \times \Omega}(\psi(y)-\psi(x)) \mathrm{d} \gamma_{k}(x, y) .
$$

We now use (69) and the Taylor formula to obtain

$$
\begin{aligned}
\int_{\Omega}\left(\rho_{k}-\rho_{k-1}\right) \psi \mathrm{d} x= & -h \int_{\Omega}<\left|\nabla_{x}\left(G^{\prime}\left(\rho_{k}\right)+V\right)\right|^{q(x)-2} \nabla_{x}\left(G^{\prime}\left(\rho_{k}\right)+V\right), \nabla \psi>\rho_{k} \mathrm{~d} x \\
& +h^{2} \int_{\Omega \times \Omega}<\left[\left[\nabla_{x}\left(G^{\prime}\left(\rho_{k}\right)+V\right)\right]^{\tau}, \nabla^{2} \psi(x+\theta(y-x))\left[\nabla_{x}\left(G^{\prime}\left(\rho_{k}\right)+V\right)\right]>\mathrm{d} \gamma_{k}(x, y),\right.
\end{aligned}
$$

where $\theta \in[0,1]$ and $\left[\nabla_{x}\left(G^{\prime}\left(\rho_{k}\right)+V\right)\right]^{\tau}$ is the transpose of $\left[\nabla_{x}\left(G^{\prime}\left(\rho_{k}\right)+V\right)\right]^{\tau}$.

Let us show that

$$
A_{k}^{h}(\psi)=h^{2} \int_{\Omega \times \Omega}<\left[\nabla_{x}\left(G^{\prime}\left(\rho_{k}\right)+V\right)\right]^{\tau}, \nabla^{2} \psi(x+\theta(y-x))\left[\nabla_{x}\left(G^{\prime}\left(\rho_{k}\right)+V\right)\right]>\mathrm{d} \gamma_{k}(x, y)
$$

converge to 0 when $h$ tends to 0 . Since $q(x)-1=(q(x) / p(x))$, we have

$$
\left|A_{k}^{h}(\psi)\right| \leq h^{2}\left\|\nabla^{2} \psi\right\|_{L^{\infty}(\Omega)} \int_{\Omega}\left|\nabla_{x}\left(G^{\prime}\left(\rho_{k}\right)+V\right)\right|^{(2 q(x) / p(x))} \rho_{k} \mathrm{~d} x .
$$

Define $\quad \Omega_{1}:=\{x \in \Omega, p(x) \geq 2\} \quad$ and $\quad \Omega_{1}:=\{x \in \Omega$, $p(x)<2\}$ :

$$
\begin{aligned}
& \int_{\Omega_{1}}\left|\nabla_{x}\left(G^{\prime}\left(\rho_{k}\right)+V\right)\right|^{(2 q(x) / p(x))} \rho_{k} \mathrm{~d} x \leq 2^{q_{+}-1}\left[\int_{\Omega}\left|\nabla_{x}\left(G^{\prime}\left(\rho_{k}\right)+V\right)\right|^{q(x)} \rho_{k} \mathrm{~d} x+1\right], \\
& \int_{\Omega_{2}}\left|\nabla_{x}\left(G^{\prime}\left(\rho_{k}\right)+V\right)\right|^{(2 q(x) / p(x))} \rho_{k} \mathrm{~d} x \leq h^{1-\frac{2}{p_{-}}}\left[\int_{\Omega}\left|\nabla_{x}\left(G^{\prime}\left(\rho_{k}\right)+V\right)\right|^{q(x)} \rho_{k} \mathrm{~d} x\right] .
\end{aligned}
$$

We use (64) and (73) in (72), and we have 


$$
\begin{aligned}
\left|A_{k}^{h}(\psi)\right| \leq & \frac{2^{p_{+}-1}}{k_{0}} h^{2}\left\|\nabla_{x} \psi\right\|_{L^{\infty}(\Omega)} \\
& +p_{+}\left(h\left\|\nabla_{x} \psi\right\|_{L^{\infty}(\Omega)}+h^{\left(2-2 / p_{-}\right)}\right) \\
& \cdot\left(\int_{\Omega} G\left(\rho_{0}\right) \mathrm{d} x-|\Omega| G\left(\frac{1}{|\Omega|}\right)+2\|\nabla\|_{L^{\infty}(\Omega)}\right) .
\end{aligned}
$$

We tend $h$ to 0 , and we conclude that $A_{k}^{h}(\psi)$ converges to 0 . Thus, the sequence $\left(\rho_{k}\right)_{k}$ is a time discretization of the nonlinear $q(x)$-Laplacian parabolic equation.

Define $\rho^{h}(t, x):[0,+\infty[\times \Omega \longrightarrow[0,+\infty[$ as follows: $\rho^{h}(t, x)=\rho_{k}(x)$ if $\left.\left.t \in\right](k-1) h, \mathrm{kh}\right]$, and $\rho^{h}(0, x)=\rho_{0}(x)$.
We prove in the next section that the sequence $\left(\rho^{h}\right)_{h}$ converges strongly to a function $\rho(t, x)$ in $L^{q(x)}([0, T] \times \Omega)$ for $0<T<\infty$ and that the nonlinear term sequence $\operatorname{div}_{x}\left(\rho^{h}\right)\left|\left(\nabla_{x}\left(G^{\prime}\left(\rho^{h}\right)\right)+V\right)\right|^{q(x)-2}\left(\nabla_{x}\left(G^{\prime}\left(\rho^{h}\right)\right)+V\right)_{h}$ converges weakly to $\operatorname{div}_{x}\left(\rho^{h}\right)\left|\left(\nabla_{x}\left(G^{\prime}\left(\rho^{h}\right)\right)+V\right)\right|^{q(x)-2}$ $\left(\nabla_{x}\left(G^{\prime}\left(\rho^{h}\right)\right)+V\right)_{h}$ in $\psi \in C_{c}^{\infty}\left(\mathbb{R}^{d}, \mathbb{R}^{d}\right)$.

3.2. Strong Convergence of $\left(\rho^{h}\right)_{h}$ and Weak Convergence of the Nonlinear Term Sequence. Here, we denote the nonlinear term sequence by $\operatorname{div}_{x}\left(\rho^{h} \omega^{h}\right)_{h}$, with $\omega^{h}:=\|\left(\nabla_{x}\right.$ $\left.\left(G^{\prime}\left(\rho^{h}\right)\right)+V\right)\left.\right|^{q(x)-2}\left(\nabla_{x}\left(G^{\prime}\left(\rho^{h}\right)\right)+V\right)$.

Since $N \leq \rho_{k} \leq M$, then $N \leq \rho^{h} \leq M$. Thus, we use (64), and we have

$$
\int_{[0, T] \times \Omega}\left|\nabla_{x} \rho^{h}\right|^{q(x)} \mathrm{d} x \leq \frac{2^{q_{+}-1}}{k_{0}}\left[\frac{p_{+}}{N}\left(\int_{\Omega} G\left(\rho_{0}\right) \mathrm{d} x-|\Omega| G\left(\frac{1}{|\Omega|}\right)+2\|V\|_{L^{\infty}(\Omega)}\right)+\|\nabla V\|_{L^{\infty}(\Omega)}^{q_{+}}\right]
$$

where $0<T<\infty$. We conclude that $\left(\rho^{h}(t, .)\right)_{h}$ is bounded in $W^{1, q(x)}(\Omega)$ for almost all $t \in[0, T]$. By using the compact injection $W^{1, q(x)}(\Omega) \hookrightarrow \hookrightarrow L^{q(x)}(\Omega)$, we deduce that $\left(\rho^{h}(t, .)\right)_{h}$ converges strongly to a function $\rho(t,$.$) in$ $L^{q(x)}(\Omega)$. Since $N \leq \rho^{h} \leq M$ and $[0, T] \times \Omega$ is bounded, we use the dominated convergence theorem to have

$$
\lim _{h \longrightarrow 0} \int_{0}^{T} \int_{\Omega}\left|\rho^{h}(t, x)-\rho(t, x)\right|^{q(x)} \mathrm{d} x \mathrm{~d} t=0 .
$$

Then, up to a subsequence, the sequence $\left(\rho^{h}\right)_{h}$ converges strongly to $\rho(t, x)$ in $L^{q(x)}([0, T] \times \Omega)$. By using (64) and $N \leq \rho^{h} \leq M$, we deduce that $\left(\nabla_{x}\left(G^{\prime}\left(\rho^{h}\right)+V\right)\right)_{h}$ is bounded in $\left[L^{q(x)}([0, T] \times \Omega)\right]^{d}$. Then, since $G^{\prime}$ is continuous and $\left(\rho^{h}\right)_{h}$ converges strongly to $\rho$ in $L^{q(x)}([0, T] \times \Omega)$, we conclude that $\left(\nabla_{x}\left(G^{\prime}\left(\rho^{h}\right)+V\right)\right)_{h}$ converges weakly to $\nabla_{x}\left(G^{\prime}(\rho)+V\right)$ in $\left[L^{q(x)}([0, T] \times \Omega)\right]^{d}$.

Note that $\left|\omega^{h}\right|^{p(x)}=\left|\nabla_{x}\left(G^{\prime}\left(\rho^{h}\right)+V\right)\right|^{q(x)}$. Then, using the fact that $\left(\nabla_{x}\left(G^{\prime}\left(\rho^{h}\right)+V\right)\right)_{h}$ is bounded in $L^{q(x)}([0, T] \times \Omega)$, we conclude that $\left(\omega^{h}\right)_{h}$ is bounded in $L^{q(x)}([0, T] \times \Omega)$. Thus, the sequence $\left(\omega^{h}\right)_{h}$ converges weakly to some $\omega \in L^{p(x)}([0, T] \times \Omega)$.

As in [7], we derive easily that $\operatorname{div}_{x}\left(\rho^{h}\right) \mid\left(\nabla_{x}\left(G^{\prime}\left(\rho^{h}\right)\right)+\right.$ $V)\left.\right|^{q(x)-2}\left(\nabla_{x}\left(G^{\prime}\left(\rho^{h}\right)\right)+V\right)_{h}$ converges weakly to $\operatorname{div}_{x}(\rho)\left|\left(\nabla_{x}\left(G^{\prime}(\rho)\right)+V\right)\right|^{q(x)-2}\left(\nabla_{x}\left(G^{\prime}(\rho)\right)+V\right)$ in $\psi \in C_{c}^{\infty}$ $\left(\mathbb{R}^{d}, \mathbb{R}^{d}\right)$.
Lemma 3. Fix $T>0$. Let $u \in C_{c}^{\infty}(\mathbb{R})$ be a test function such that suppu $\subset[-T, T]$. We have

$$
\begin{gathered}
\lim _{h \rightarrow 0} \int_{[0, T] \times \Omega}<\rho^{h} \omega^{h}, \nabla_{x}\left(G^{\prime}\left(\rho^{h}\right)+V\right)>u(t) \mathrm{d} t \mathrm{~d} x \\
=\int_{[0, T] \times \Omega}<\rho \omega, \nabla_{x}\left(G^{\prime}\left(\rho^{h}\right)+V\right)>u(t) \mathrm{d} t \mathrm{~d} x .
\end{gathered}
$$

Proof 3. The proof of this lemma is achieved via the following three claims:

Claim 1:

$$
\begin{gathered}
\liminf _{h \longrightarrow 0} \int_{[0, T] \times \Omega} \rho^{h}<\omega^{h}, \nabla_{x}\left(G^{\prime}\left(\rho^{h}\right)+V\right)>u(t) \mathrm{d} t \mathrm{~d} x \\
\geq \int_{[0, T] \times \Omega} \rho<\omega, \nabla_{x}\left(G^{\prime}\left(\rho^{h}\right)+V\right)>u(t) \mathrm{d} t \mathrm{~d} x .
\end{gathered}
$$

Proof 4. Since $\rho^{h} \geq 0, u \geq 0$, and $v \longmapsto|v|^{q(x)-2} v$ is monotone for all $x$ fixed in $\Omega$, we have

$$
\begin{gathered}
\int_{[0, T] \times \Omega} \rho^{h}<\omega^{h}-\left|\nabla_{x}\left(G^{\prime}(\rho)+V\right)\right|^{q(x))-2} \nabla_{x}\left(G^{\prime}(\rho)+V\right), \\
\nabla_{x}\left(G^{\prime}\left(\rho^{h}\right)+V\right)-\nabla_{x}\left(G^{\prime}(\rho)+V\right)>u(t) \mathrm{d} t \mathrm{~d} x \geq 0 .
\end{gathered}
$$


Using (81), we have

$$
\begin{aligned}
& \int_{[0, T] \times \Omega}<\rho^{h} \omega^{h}, \nabla_{x}\left(G^{\prime}\left(\rho^{h}\right)+V\right)>u(t) \mathrm{d} t \mathrm{~d} x \geq \int_{[0, T] \times \Omega}<\rho^{h} \omega^{h}, \nabla_{x}\left(G^{\prime}(\rho)+V\right)>u(t) \mathrm{d} t \mathrm{~d} x+ \\
& \int_{[0, T] \times \Omega}<\rho^{h}\left|\nabla_{x}\left(G^{\prime}(\rho)+V\right)\right|^{q(x)-2} \nabla_{x}\left(G^{\prime}(\rho)+V\right), \nabla_{x}\left(G^{\prime}\left(\rho^{h}\right)+V\right)-\nabla_{x}\left(G^{\prime}(\rho)+V\right)>u(t) \mathrm{d} t \mathrm{~d} x .
\end{aligned}
$$

Since $\left(\rho^{h}\right)_{h}$ converges strongly to $\rho$ in $L^{q(x)}([0, T] \times \Omega)$, $\left(\nabla_{x}\left(G^{\prime}\left(\rho^{h}\right)+V\right)\right)_{h}$ converges weakly to $\nabla_{x}\left(G^{\prime}(\rho)+V\right)$ in

$$
\begin{aligned}
& \lim \inf _{h \longrightarrow 0} \int_{[0, T] \times \Omega}<\rho^{h} \omega^{h}, \nabla_{x}\left(G^{\prime}(\rho)+V\right)>u(t) \mathrm{d} t \mathrm{~d} x=\int_{[0, T] \times \Omega}<\rho \omega, \nabla_{x}\left(G^{\prime}(\rho)+V\right)>u(t) \mathrm{d} t \mathrm{~d} x, \\
& \lim \inf _{h \longrightarrow 0} \int_{[0, T] \times \Omega}<\rho^{h}\left|\nabla_{x}\left(G^{\prime}(\rho)+V\right)\right|^{q(x)-2} \nabla_{x}\left(G^{\prime}(\rho)+V\right), \nabla_{x}\left(G^{\prime}\left(\rho^{h}\right)+V\right)-\nabla_{x}\left(G^{\prime}(\rho)+V\right)>u(t) \mathrm{d} t \mathrm{~d} x=0 .
\end{aligned}
$$

We now use (83) and (84) in (82) to conclude the proof of claim 1 .

\section{Claim 2:}

$$
\begin{aligned}
& \lim \sup _{h \longrightarrow 0} \int_{[0, T] \times \Omega}<\rho^{h} \omega^{h}, \nabla_{x}\left(G^{\prime}\left(\rho^{h}\right)+V\right)>u(t) \mathrm{d} t \mathrm{~d} x \leq \int_{\Omega}\left[\rho_{0} G^{\prime}\left(\rho_{0}\right)-G^{*}\left(G^{\prime}\left(\rho_{0}\right)\right)\right] u(0) \mathrm{d} x+ \\
& \int_{[0, T] \times \Omega}\left[\rho G^{\prime}(\rho)-G^{*}\left(G^{\prime}\left(\rho^{h}\right)\right)\right] u^{\prime}(t) \mathrm{d} t \mathrm{~d} x+\int_{[0, T] \times \Omega} \rho V u^{\prime}(t) \mathrm{d} t \mathrm{~d} x+\int_{\Omega} \rho_{0} V u(0) \mathrm{d} x .
\end{aligned}
$$

Proof 5. We use the descent algorithm $\left(P_{k}\right)$ and (70) to obtain

$$
\begin{gathered}
\int_{\Omega}\left(G\left(\rho_{k-1}\right)+\rho_{k-1} V\right) \mathrm{d} x-\int_{\Omega}\left(G\left(\rho_{k}\right)+\rho_{k} V\right) \mathrm{d} x \\
\geq h \int_{\Omega} \frac{\left|\nabla_{x}\left(G^{\prime}\left(\rho_{k}\right)+V\right)\right|^{q(x)}}{p(x)} \rho_{k} \mathrm{~d} x .
\end{gathered}
$$

$\frac{1}{h} \sum_{k=1}^{(T / h)} \int_{h(k-1)}^{h k}\left[\int_{\Omega}\left(G\left(\rho_{k-1}\right)+\rho_{k-1} V\right) \mathrm{d} x-\int_{\Omega}\left(G\left(\rho_{k}\right)+\rho_{k} V\right) \mathrm{d} x\right] u(t) \mathrm{d} t \geq \int_{0}^{T} \int_{\Omega} \frac{<\omega_{k}, \nabla_{x}\left(G^{\prime}\left(\rho_{k}\right)+V\right)>}{p(x)} \rho_{k} u(t) \mathrm{d} t \mathrm{~d} x$

We use $p(x) \leq p_{+}$and $\tau=\left(h / p_{+}\right)$in (87), and we have

$\frac{1}{h} \sum_{k=1}^{(T / h)} \int_{h(k-1)}^{h k}\left[\int_{\Omega}\left(G\left(\rho_{k-1}\right)+\rho_{k-1} V\right) \mathrm{d} x-\int_{\Omega}\left(G\left(\rho_{k}\right)+\rho_{k} V\right) \mathrm{d} x\right] u(t) \mathrm{d} t \geq \int_{0}^{T} \int_{\Omega}<\omega^{\tau}, \nabla_{x}\left(G^{\prime}\left(\rho^{\tau}\right)+V\right)>\rho^{\tau} u(t) \mathrm{d} t \mathrm{~d} x$

where $\rho^{\tau}$ is defined as follows:

$$
\left.\left.\rho^{\tau}(t, x)=\rho_{k}(x), \quad \text { if } t \in\right] \tau(k-1), \tau k\right], \rho^{\tau}(0, x)=\rho_{0}(x),
$$


and $\omega^{\tau}=\left|\nabla_{x}\left(G^{\prime}\left(\rho^{\tau}\right)+V\right)\right|^{q(x)-2} \nabla_{x}\left(G^{\prime}\left(\rho^{\tau}\right)+V\right)$.

$$
\begin{aligned}
& \frac{1}{\tau} \sum_{k=1}^{(T / \tau)} \int_{\tau(k-1)}^{\tau k}\left[\int_{\Omega}\left(G\left(\rho_{k-1}\right)+\rho_{k-1} V\right) \mathrm{d} x-\int_{\Omega}\left(G\left(\rho_{k}\right)+\rho_{k} V\right) \mathrm{d} x\right] u(t) \mathrm{d} t=\frac{1}{\tau} \int_{0}^{\tau} \int_{\Omega}\left(G\left(\rho_{0}\right)+\rho_{0} V\right) u(t) \mathrm{d} t \mathrm{~d} x \\
& \quad+\int_{0}^{T-\tau}\left(\frac{u(t+\tau)-u(t)}{\tau}\right) \int_{\Omega}\left(G\left(\rho^{\tau}\right)+\rho^{\tau} V\right) \mathrm{d} t \mathrm{~d} x .
\end{aligned}
$$

Recalling inequalities (88) and (89), we get

$$
\begin{aligned}
\int_{0}^{T} \int_{\Omega}<\omega^{\tau}, \nabla_{x}\left(G^{\prime}\left(\rho^{\tau}\right)+V\right)>\rho^{\tau} u(t) \mathrm{d} t \mathrm{~d} x \leq & \frac{1}{\tau} \int_{0}^{\tau} \int_{\Omega}\left(G\left(\rho_{0}\right)+\rho_{0} V\right) u(t) \mathrm{d} t \mathrm{~d} x \\
& \left.+\int_{0}^{T-\tau}\left(\frac{u(t+\tau)-u(t)}{\tau}\right)\right) \int_{\Omega}\left(G^{\prime}\left(\rho^{\tau}\right)+\rho^{\tau} V\right) \mathrm{d} t \mathrm{~d} x .
\end{aligned}
$$

We tend $\tau$ toward 0 , and we obtain

$\lim \sup _{\tau \longrightarrow 0} \int_{[0, T] \times \Omega}<\rho^{\tau} \omega^{\tau}, \nabla_{x}\left(G^{\prime}\left(\rho^{\tau}\right)+V\right)>u(t) \mathrm{d} t \mathrm{~d} x \leq \int_{\Omega}\left(G\left(\rho_{0}\right)+\rho_{0} V\right) u(0) \mathrm{d} x+\int_{[0, T] \times \Omega}\left(G^{\prime}\left(\rho^{\tau}\right)+\rho V\right) u^{\prime}(t) \mathrm{d} t \mathrm{~d} x$

From the strict convexity of $G \in C^{1}(] 0, \infty[)$, we derive that

$$
\begin{aligned}
G\left(\rho_{0}\right) & =\rho_{0} G^{\prime}\left(\rho_{0}\right)-G^{*}\left(G^{\prime}\left(\rho_{0}\right)\right), \text { and } G(\rho) \\
& =\rho G^{\prime}(\rho)-G^{*}(G(\rho)),
\end{aligned}
$$

where $G^{*}$ is a Legendre transform of $G$.

We conclude the proof of claim 2 by using (92) in (93). Claim 3:

$$
\begin{aligned}
\int_{[0, T] \times \Omega}<\rho \omega, \nabla_{x}\left(G^{\prime}(\rho)+V\right)>u(t) \mathrm{d} t \mathrm{~d} x \geq & \int_{\Omega}\left[\rho_{0} G^{\prime}\left(\rho_{0}\right)-G^{*}\left(G^{\prime}\left(\rho_{0}\right)\right)\right] u(0) \mathrm{d} x+\int_{[0, T] \times \Omega}\left[\rho G(\rho)-G^{*}\left(G^{\prime}(\rho)\right)\right] u^{\prime}(t) \mathrm{d} t \mathrm{~d} x \\
& +\int_{[0, T] \times \Omega} \rho V u^{\prime}(t) \mathrm{d} t \mathrm{~d} x+\int_{\Omega} \rho_{0} V u(0) \mathrm{d} x .
\end{aligned}
$$

Proof 6. Define $\quad \psi(t, x)=\left(G^{\prime}(\rho)+V\right) u(t), \quad$ for $\quad \psi \in W^{1, q(x)}([0, T] \times \Omega)$, and then we approximate $\psi$ by $(t, x) \in[0, T] \times \Omega$. $C_{c}^{\infty}\left(\mathbb{R} \times \mathbb{R}^{d}\right)$-functions, and (70) becomes

$$
\frac{1}{h} \sum_{k=1}^{(T / h)} \int_{h(k-1)}^{h k} \int_{\Omega}\left(\rho_{k-1}-\rho_{k}\right) \psi(t, x) \mathrm{d} t \mathrm{~d} x-\int_{[0, T] \times \Omega}<\rho^{h} \omega^{h},\left(\nabla_{x}\left(G^{\prime}\right)(\rho)+V\right)>u(t) \mathrm{d} t \mathrm{~d} x=o(h),
$$


where $o(h)$ converges to 0 when $h$ tends to 0 . As in (89), we have

$$
\frac{1}{h} \sum_{k=1}^{(T / h)} \int_{h(k-1)}^{h k} \int_{\Omega}\left(\rho_{k-1}-\rho_{k}\right) \psi(t, x) \mathrm{d} t \mathrm{~d} x=\frac{1}{h} \int_{0}^{h} \int_{\Omega} \rho_{0} \psi(t, x) \mathrm{d} t \mathrm{~d} x+\int_{h}^{T} \int_{\Omega} \rho^{h} \partial_{t}^{-h} \psi(t, x) \mathrm{d} t \mathrm{~d} x
$$

where $\partial_{t}^{-h} \psi(t, x)=(\psi(t, x)-\psi(t-h, x) / h)$.

Using (96), (97) becomes

$$
\frac{1}{h} \int_{0}^{h} \int_{\Omega} \psi(t, x) \rho_{0} \mathrm{~d} t \mathrm{~d} x+\int_{h}^{T} \int_{\Omega} \rho^{h} \partial_{t}^{-h} \psi(t, x) \mathrm{d} t \mathrm{~d} x-\int_{[0, T] \times \Omega}<\rho^{h} \omega^{h}, \nabla_{x}\left(\left(G^{\prime}\right)(\rho)+V\right)>u(t) \mathrm{d} t \mathrm{~d} x=o(h) .
$$

We tend $h$ to 0 in (98) and use the fact that $\rho^{h}$ converges strongly to $\rho$ in $L^{q(x)}([0, T] \times \Omega)$ and $\omega^{h}$ weakly to $\omega$ in $L^{q(x)}([0, T] \times \Omega)$ to get

$$
\int_{\Omega} \rho_{0}\left[G^{\prime}\left(\rho_{0}\right)+V\right] u(0) \mathrm{d} x+\lim _{h \longrightarrow 0} \int_{0}^{T} \int_{\Omega} \rho \partial_{t}^{-h} \psi(t, x) \mathrm{d} t \mathrm{~d} x-\int_{[0, T] \times \Omega}<\rho \omega, \nabla_{x}\left(\left(G^{\prime}\right)(\rho)+V\right)>u(t) \mathrm{d} t \mathrm{~d} x=0
$$

By using

$$
\begin{gathered}
\rho \partial^{-h} \psi(t, x)=\rho G^{\prime}(\rho) \partial_{t}^{-h} u(t)+\rho V \partial_{t}^{-h} u(t)+\frac{1}{h} \rho u(t-h)\left[G^{\prime}(\rho)-G^{\prime}(\rho(t-h, x))\right] \\
\rho\left[G^{\prime}(\rho)-G^{\prime}(\rho(t-h, x))\right] \geq G^{*}\left(G^{\prime}(\rho)\right)-G^{*}\left(G^{\prime}(\rho(t-h, x))\right),
\end{gathered}
$$

we have

$$
\begin{aligned}
& \rho \partial^{-h} \psi(t, x) \geq \rho G^{\prime}(\rho) \partial_{t}^{-h} u(t)+\rho V \partial_{t}^{-h} u(t)+\frac{1}{h} u(t-h)\left[G^{*}\left(G^{\prime}(\rho)\right)-G^{*}\left(G^{\prime}(\rho(t-h, x))\right)\right] \\
& =\left[\rho G^{\prime}(\rho)-G^{*}\left(G^{\prime}(\rho)\right)\right] \partial_{t}^{-h} u(t)+\rho V \partial_{t}^{-h} u(t)+\frac{1}{h}\left[G^{*}\left(G^{\prime}(\rho)\right) u(t)-G^{*}\left(G^{\prime}(\rho(t-h, x))\right) u(t-h)\right] .
\end{aligned}
$$

Next, after integration of the inequality above, we have

$$
\begin{aligned}
& \int_{0}^{T} \int_{\Omega} \rho \partial^{-h} \psi(t, x) \mathrm{d} t \mathrm{~d} x \geq \int_{0}^{T} \int_{\Omega}\left[\rho G^{\prime}(\rho)-G^{*}\left(G^{\prime}(\rho)\right)\right] \partial_{t}^{-h} u(t) \mathrm{d} t \mathrm{~d} x \\
& +\int_{0}^{T} \int_{\Omega} \rho V \partial_{t}^{-h} u(t) \mathrm{d} t \mathrm{~d} x+\frac{1}{h} \int_{0}^{T} \int_{\Omega}\left[G^{*}\left(G^{\prime}(\rho)\right) u(t)-G^{*}\left(G^{\prime}(\rho(t-h, x))\right) u(t-h)\right] \mathrm{d} t \mathrm{~d} x .
\end{aligned}
$$


Note that

$$
\begin{aligned}
& \frac{1}{h} \int_{0}^{T} \int_{\Omega}\left[G^{*}\left(G^{\prime}(\rho)\right) u(t)-G^{*}\left(G^{\prime}(\rho(t-h, x))\right) u(t-h)\right] \mathrm{d} t \mathrm{~d} x \\
& =\frac{1}{h} \int_{0}^{T} \int_{\Omega} G^{*}\left(G^{\prime}(\rho)\right) u(t) \mathrm{d} t \mathrm{~d} x-\frac{1}{h} \int_{0}^{T} \int_{\Omega}\left[G^{*}\left(G^{\prime}(\rho(t-h, x))\right) u(t-h)\right] \mathrm{d} t \mathrm{~d} x, \\
& \quad \frac{1}{h} \int_{0}^{T} \int_{\Omega}\left[G^{*}\left(G^{\prime}(\rho(t-h, x))\right) u(t-h)\right] \mathrm{d} t \mathrm{~d} x=\frac{1}{h} \sum_{k=1}^{(T / h)} \int_{h(k-1)}^{h k} \int_{\Omega}\left[G^{*}\left(G^{\prime}(\rho(t-h, x))\right) u(t-h)\right] \mathrm{d} t \mathrm{~d} x
\end{aligned}
$$

Using the change of variable $s=t-h$ in (105), we obtain

$$
\int_{h(k-1)}^{h k} \int_{0}^{T} \int_{\Omega}\left[G^{*}\left(G^{\prime}(\rho(t-h, x))\right) u(t-h)\right] \mathrm{d} t \mathrm{~d} x=\int_{h(k-2)}^{h(k-1)} \int_{0}^{T} \int_{\Omega}\left[G^{*}\left(G^{\prime}(\rho(t-h, x))\right) u(t-h)\right] \mathrm{d} t \mathrm{~d} x, \quad \text { for all } k \geq 2 .
$$

We combine (106) and (105) to deduce that

$$
\begin{aligned}
& \frac{1}{h} \int_{0}^{T} \int_{\Omega} G^{*}\left(G^{\prime}(\rho(t-h, x))\right) u(t-h) \mathrm{d} t \mathrm{~d} x \\
& \quad=\frac{1}{h} \int_{0}^{h} \int_{\Omega}\left[G^{*}\left(G^{\prime}(\rho(t-h, x))\right) u(t-h)\right] \mathrm{d} t \mathrm{~d} x+\frac{1}{h} \sum_{k=2}^{(T / h)} \int_{h(k-2)}^{h(k-1)} \int_{\Omega} G^{*}\left(G^{\prime}(\rho(t-h, x))\right) u(t) \mathrm{d} t \mathrm{~d} x \\
& \quad=\frac{1}{h} \int_{0}^{h} \int_{\Omega} G^{*}\left(G^{\prime}(\rho(t-h, x))\right) u(t-h) \mathrm{d} t \mathrm{~d} x+\frac{1}{h} \int_{0}^{T-h} \int_{\Omega} G^{*}\left(G^{\prime}(\rho(t-h, x))\right) u(t) \mathrm{d} t \mathrm{~d} x
\end{aligned}
$$

Now, we use (107) in (108) to derive

$$
\begin{aligned}
& \frac{1}{h} \int_{0}^{T} \int_{\Omega}\left[G^{*}\left(G^{\prime}(\rho)\right) u(t)-G^{*}\left(G^{\prime}(\rho(t-h, x))\right) u(t-h)\right] \mathrm{d} t \mathrm{~d} x \\
& \quad=-\frac{1}{h} \int_{0}^{h} \int_{\Omega}\left[G^{*}\left(G^{\prime}(\rho(t-h, x))\right) u(t-h)\right]+\frac{1}{h} \int_{T-h}^{T} \int_{\Omega} G^{*}(\rho(t, x)) u(t) \mathrm{d} t \mathrm{~d} x
\end{aligned}
$$

So, (103) becomes

$$
\begin{aligned}
& \int_{0}^{T} \int_{\Omega} \rho \partial^{-h} \psi(t, x) \mathrm{d} t \mathrm{~d} x \geq \int_{0}^{T} \int_{\Omega}\left[\rho G^{\prime}(\rho)-G^{*}\left(G^{\prime}(\rho)\right)\right] \partial_{t}^{-h} u(t) \mathrm{d} t \mathrm{~d} x \\
& +\int_{0}^{T} \int_{\Omega} \rho V \partial_{t}^{-h} u(t) \mathrm{d} t \mathrm{~d} x-\frac{1}{h} \int_{0}^{h} \int_{\Omega} G^{*}\left(G^{\prime}(\rho)\right) u(t-h) \mathrm{d} t \mathrm{~d} x+\frac{1}{h} \int_{T-h}^{T} \int_{\Omega} G^{*}\left(G^{\prime}(\rho(t, x))\right) u(t) \mathrm{d} t \mathrm{~d} x
\end{aligned}
$$


Since $\operatorname{supp}(u) \subset[-T, T]$, then

$$
\lim _{h \longrightarrow 0} \frac{1}{h} \int_{T-h}^{T} \int_{\Omega} G^{*}\left(G^{\prime}(\rho(t, x))\right) u(t) \mathrm{d} t \mathrm{~d} x=\int_{\Omega} G^{*}\left(G^{\prime}(\rho(T, x))\right) u(T) \mathrm{d} x=0 .
$$

We deduce that

$\lim _{h \longrightarrow 0} \int_{0}^{T} \int_{\Omega} \rho \partial^{-h} \psi(t, x) \mathrm{d} t \mathrm{~d} x \geq \int_{0}^{T} \int_{\Omega}\left[\rho G^{\prime}(\rho)-G^{*}\left(G^{\prime}(\rho)\right)\right] u^{\prime}(t) \mathrm{d} t \mathrm{~d} x+\int_{0}^{T} \int_{\Omega} \rho V u^{\prime}(t) \mathrm{d} t \mathrm{~d} x-\int_{\Omega} G^{*}\left(G^{\prime}\left(\rho_{0}\right)\right) u(0) \mathrm{d} x$.

Now, we combine (99) and (111) to obtain

$$
\begin{gathered}
\int_{0}^{T} \int_{\Omega}\left[\rho G^{\prime}(\rho)-G^{*}\left(G^{\prime}(\rho)\right)\right] u^{\prime}(t) \mathrm{d} t \mathrm{~d} x+\int_{0}^{T} \int_{\Omega} \rho V u^{\prime}(t) \mathrm{d} t \mathrm{~d} x \\
-\int_{\Omega} G^{*}\left(G^{\prime}\left(\rho_{0}\right)\right) u(0) \mathrm{d} x+\int_{\Omega} \rho_{0}\left[G^{\prime}\left(\rho_{0}\right)+V\right] u(0) \mathrm{d} x \\
-\int_{[0, T] \times \Omega}<\rho \omega, \nabla_{x}\left(\left(G^{\prime}\right)(\rho)+V\right)>u(t) \mathrm{d} t \mathrm{~d} x \leq 0 .
\end{gathered}
$$

Finally, we conclude that

$$
\begin{aligned}
& \int_{0}^{T} \int_{\Omega}\left[\rho G^{\prime}(\rho)-G^{*}\left(G^{\prime}(\rho)\right)\right] u^{\prime}(t) \mathrm{d} t \mathrm{~d} x+\int_{0}^{T} \int_{\Omega} \rho V u^{\prime}(t) \mathrm{d} t \mathrm{~d} x+\int_{\Omega} \rho_{0} V u(0) \mathrm{d} x \\
& \quad+\int_{\Omega}\left[\rho_{0} G^{\prime}\left(\rho_{0}\right)-G^{*}\left(G^{\prime}\left(\rho_{0}\right)\right)\right] u(0) \mathrm{d} x \\
& \int_{[0, T] \times \Omega}<\rho \omega, \nabla_{x}\left(\left(G^{\prime}\right)(\rho)+V\right)>u(t) \mathrm{d} t \mathrm{~d} x .
\end{aligned}
$$

This completes the proof of claim 3.

Now, we are ready to show that the sequence $\left(\operatorname{div}_{x}\left(\rho^{h} \omega^{h}\right)\right)_{h}$ converges weakly to $\operatorname{div}_{x}\left(\rho \mid \nabla_{x}\left(G^{\prime}(\rho)+\right.\right.$ $V) \mid)^{q(x)-2} \nabla_{x}\left(G^{\prime}(\rho)+V\right) \quad$ in $\quad\left[C_{c}^{\infty}\left(\mathbb{R} \times \mathbb{R}^{d}\right)\right] \prime$. Let $\psi \in C_{c}^{\infty}\left(\mathbb{R}^{d}\right)$ and $u \in C_{c}^{\infty}(\mathbb{R})$ be some positive functions such that $\operatorname{supp}(u) \subset[-T, T]$, with $0<T<\infty$. For $\epsilon \neq 0$, we define $\psi_{\epsilon}(t, x)=G^{\prime}(\rho)+V-\epsilon \psi$, and then

$$
\int_{[0, T] \times \Omega} \rho^{h}<\omega^{h}-\left|\nabla_{x} \psi_{\epsilon}\right|^{q(x)-2} \nabla_{x} \psi_{\epsilon}, \nabla_{x}\left(G^{\prime}\left(\rho^{h}\right)+V\right)-\nabla_{x} \psi_{\epsilon}>u(t) \mathrm{d} t \mathrm{~d} x \geq 0 .
$$

So, we obtain

$$
\begin{gathered}
\int_{[0, T] \times \Omega} \rho^{h}<\omega^{h}, \nabla_{x}\left(G^{\prime}\left(\rho^{h}\right)+V\right)<u(t) \mathrm{d} t \mathrm{~d} x-\int_{[0, T] \times \Omega} \rho^{h}<\omega^{h}, \nabla_{x} \psi_{\epsilon}>u(t) \mathrm{d} t \mathrm{~d} x \\
+\int_{[0, T] \times \Omega} \rho^{h}<\left|\nabla_{x} \psi_{\epsilon}\right|^{q(x)-2} \nabla_{x} \psi_{\epsilon}, \nabla_{x} \psi_{\epsilon}-\nabla_{x}\left(G^{\prime}\left(\rho^{h}\right)+V\right)>u(t) \mathrm{d} t \mathrm{~d} x \geq 0 .
\end{gathered}
$$


We use Lemma 3 and we tend $h$ to 0 in (115), and then we get

$$
\begin{gathered}
\int_{[0, T] \times \Omega} \rho<\omega^{h}, \nabla_{x}\left(G^{\prime}\left(\rho^{h}\right)+V\right)<u(t) \mathrm{d} t \mathrm{~d} x-\int_{[0, T] \times \Omega} \rho<\omega^{h}, \nabla_{x} \psi_{\epsilon}>u(t) \mathrm{d} t \mathrm{~d} x \\
+\int_{[0, T] \times \Omega} \rho<\left|\nabla_{x} \psi_{\epsilon}\right|^{q(x)-2} \nabla_{x} \psi_{\epsilon}, \nabla_{x} \psi_{\epsilon}-\nabla_{x}\left(G^{\prime}\left(\rho^{h}\right)+V\right)>u(t) \mathrm{d} t \mathrm{~d} x \geq 0 .
\end{gathered}
$$

So,

Dividing (116) by $\epsilon>0$ and tending $\epsilon$ to 0 , we reach

$\int_{[0, T] \times \Omega} \rho<\omega-\left|\nabla_{x} \psi_{\epsilon}\right|^{q(x)-2} \nabla_{x} \psi_{\epsilon}, \epsilon \nabla_{x} \psi>u(t) \mathrm{d} t \mathrm{~d} x \geq 0$.

$$
\int_{[0, T] \times \Omega} \rho<\omega-\left|\nabla_{x}\left(G^{\prime}(\rho) V\right)\right|^{q(x)-2} \nabla_{x}\left(G^{\prime}(\rho) V\right), \nabla_{x} \psi>u(t) \mathrm{d} t \mathrm{~d} x \geq 0 .
$$

By replacing $\psi$ by $-\psi$, we have

$$
\int_{[0, T] \times \Omega} \rho<\omega-\left|\nabla_{x}\left(G^{\prime}(\rho) V\right)\right|^{q(x)-2} \nabla_{x}\left(G^{\prime}(\rho) V\right), \nabla_{x} \psi>u(t) \mathrm{d} t \mathrm{~d} x=0,
$$

for all test functions $\psi \in C_{c}^{\infty}\left(\mathbb{R}^{d}\right)$ and $u \in C_{c}^{\infty}(\mathbb{R})$ such that $\operatorname{supp}(u) \subset[-T, T]$.

Thus, we conclude that $\left(\operatorname{div}_{x}\left(\rho^{h} \omega^{h}\right)\right)_{h}$ converges weakly to $\operatorname{div}_{x}\left(\rho\left|\nabla_{x}\left(G^{\prime}(\rho) V\right)\right|^{q(x)-2} \nabla_{x}\left(G^{\prime}(\rho) V\right)\right)$ in $\left[C_{c}^{\infty}(\mathbb{R} \times\right.$ $\left.\left.\mathbb{R}^{d}\right)\right]$.

\subsection{Proof of the Theorem of the Laplacian Parabolic Equation.} Here, we use the strong convergence of the sequence $\left(\rho^{h}\right)_{h}$ to $\rho$ in $L^{q(x)}([0, T] \times \Omega)$ and the weak convergence of the nonlinear term $\left(\operatorname{div}_{x}\left(\rho^{h} \omega^{h}\right)\right)_{h}$ to $\operatorname{div}_{x}\left(\rho\left|\nabla_{x}\left(G^{\prime}(\rho) V\right)\right|^{q(x)-2} \nabla_{x}\left(G^{\prime}(\rho) V\right)\right)$ in $\left[C_{c}^{\infty}\left(\mathbb{R} \times \mathbb{R}^{d}\right)\right] ı$, and we establish that $\rho(t, x)$ is a weak solution of the $q(x)$ Laplacian parabolic equation. Let $\psi \in C_{c}^{\infty}\left(\mathbb{R} \times \mathbb{R}^{d}\right)$ be a test function such that $\operatorname{supp} \psi(., x) \subset[-T, T]$. By using (71), we obtain

$$
\begin{gathered}
\frac{1}{h} \sum_{k=1}^{(T / h)} \int_{[h(k-1), \mathrm{hk}] \times \Omega}\left(\rho_{k-1}-\rho_{k}\right) \psi(t, x) \mathrm{d} t \mathrm{~d} x \\
-\int_{[0, T] \times \Omega} \rho^{h}<\omega^{h}, \nabla_{x} \psi>\mathrm{d} t \mathrm{~d} x=0(h),
\end{gathered}
$$

where $o(h)$ tends to 0 when $h$ tends to 0 .

Note that

$$
\begin{aligned}
& \frac{1}{h} \sum_{k=1}^{(T / h)} \int_{[h(k-1), \mathrm{hk}] \times \Omega}\left(\rho_{k-1}-\rho_{k}\right) \psi(t, x) \mathrm{d} t \mathrm{~d} x \\
& =\frac{1}{h} \int_{0}^{h} \int_{\Omega} \rho_{0} \psi(t, x) \mathrm{d} t \mathrm{~d} x+\int_{h}^{T-h} \int_{\Omega} \rho^{h} \partial_{t}^{-h} \psi(t, x) \mathrm{d} t \mathrm{~d} x .
\end{aligned}
$$

Then, (120) becomes

$$
\begin{gathered}
\frac{1}{h} \int_{0}^{h} \int_{\Omega} \rho_{0} \psi(t, x) \mathrm{d} t \mathrm{~d} x+\int_{h}^{T-h} \int_{\Omega} \rho^{h} \partial_{t}^{-h} \psi(t, x) \mathrm{d} t \mathrm{~d} x \\
-\int_{[0, T] \times \Omega} \rho^{h}<\omega^{h}, \quad \nabla_{x} \psi>d t \mathrm{~d} x=o(h) .
\end{gathered}
$$

Finally, we tend $h$ to 0 in (122), and we use the fact that $\rho^{h}$ converges strongly to $\rho$ and that $\left(\operatorname{div}_{x}\left(\rho^{h} \omega^{h}\right)\right)_{h}$ converges weakly to $\operatorname{div}_{x}\left(\rho\left|\nabla_{x}\left(G^{\prime}(\rho) V\right)\right|^{q(x)-2} \nabla_{x}\left(G^{\prime}(\rho) V\right)\right)$ :

$$
\begin{aligned}
& \int_{\Omega} \rho_{0} \psi(0, x) \mathrm{d} x+\int_{[0, T] \times \Omega} \rho \frac{\partial \psi}{\partial t} \mathrm{~d} t \mathrm{~d} x-\int_{[0, T] \times \Omega} \rho \\
& <\left|\nabla_{x}\left(G^{\prime}(\rho)+V\right)\right|^{q(x)-2} \nabla_{x}\left(G^{\prime}(\rho)+V\right), \nabla_{x} \psi>\mathrm{d} t \mathrm{~d} x=0 .
\end{aligned}
$$

We deduce that 


$$
\begin{gathered}
\int_{[0, T] \times \Omega} \rho<\frac{\partial \psi}{\partial t}-\left|\nabla_{x}\left(G^{\prime}(\rho)+V\right)\right|^{q(x)-2} \nabla_{x}\left(G^{\prime}(\rho)+V\right), \\
\nabla_{x} \psi>\mathrm{d} t \mathrm{~d} x=-\int_{\Omega} \rho_{0} \psi(0, x) \mathrm{d} x .
\end{gathered}
$$

It follows that $\rho(t, x)$ is a weak solution of a nonlinear $q(x)$-Laplacian parabolic equation.

\section{Asymptotic Behavior}

In this section, we give the proof of Theorem 2 which is derived from the following three lemmas.

Lemma 4. Let $\rho_{1}$ and $\rho_{2}$ be two probability densities on $\Omega$. Assume that $V$ satisfies $\left(H_{V}^{2}\right)$. If $T_{\#}^{+} \rho_{1}=\rho_{2}$ is the solution of the Monge problem

$$
\left(M_{+}\right): \inf _{T_{\#}^{+} \rho_{1}=\rho_{2}} \int_{\Omega}\left|x-T^{+}\right|(x)^{p_{+}} \rho_{1} \mathrm{~d} x,
$$

then

$$
\begin{aligned}
& E\left(\rho_{1}\right)-E\left(\rho_{2}\right)+\frac{\lambda}{2^{p_{+}}} W_{\left.p^{(} .\right)}^{p_{+}}\left(\rho_{1}, \rho_{2}\right) \\
& \quad \leq \int_{\Omega}<x-T^{+}(x), \nabla_{x}\left(G^{\prime}\left(\rho_{1}\right)+V\right)>\rho_{1} \mathrm{~d} x .
\end{aligned}
$$

In particular, if $\rho_{\infty} \in P(\Omega) \quad$ satisfies $\rho_{\infty} \nabla_{x}\left(G^{\prime}\left(\rho_{\infty}\right)+V\right)=0$, then

$$
W_{p(.)}^{p_{+}}\left(\rho, \rho_{\infty}\right) \leq \frac{2^{p_{+}}}{\lambda}\left[E(\rho)-E\left(\rho_{\infty}\right)\right],
$$

for all $\rho \in P(\Omega)$.

Here, $W_{p(.)}$ is the $p($.)-Wasserstein distance defined in (5).

Proof 7. Let $\rho_{1}, \rho_{2} \in P(\Omega)$. Fix $p_{+}=\sup _{\Omega} p(x)>1$. It is known from the work in [8] that the Monge problem

$$
\left(M_{+}\right): \inf _{T_{\#}^{+} \rho_{1}=\rho_{2}} \int_{\Omega}\left|x-T^{+}\right|(x)^{p_{+}} \rho_{1} \mathrm{~d} x,
$$

admits a unique solution $T^{+}$. Therefore, since $G$ and $V$ satisfy, respectively, $\left(H_{G}\right)$ and $\left(H_{V}^{2}\right)$, then

$$
\begin{aligned}
& E\left(\rho_{1}\right)-E\left(\rho_{2}\right)+\lambda W_{P_{+}}^{p_{+}}\left(\rho_{1}, \rho_{2}\right) \\
& \quad \leq \int_{\Omega}<x-T^{+}, \nabla_{x}\left(G^{\prime}\left(\rho_{1}\right)+V\right)>\rho_{1} \mathrm{~d} x,
\end{aligned}
$$

where $\int_{\Omega}\left|x-T^{+}\right|^{p_{+}} \rho_{1} \mathrm{~d} x=W_{p_{+}}^{p_{+}}\left(\rho_{1}, \rho_{2}\right)$ and $W_{p_{+}}$is the $p_{+}$-Wasserstein distance.

The continuous injection $L_{\rho_{1}}^{p_{+}}(\Omega) \hookrightarrow L_{\rho_{1}}^{p(.)}(\Omega)$ implies that

$$
W_{p(.)}\left(\rho_{1}, \rho_{2}\right) \leq 2 W_{p_{+}}\left(\rho_{1}, \rho_{2}\right) \text {. }
$$

So, we use (129) and (128) to derive

$$
\begin{aligned}
& E\left(\rho_{1}\right)-E\left(\rho_{2}\right)+\frac{\lambda}{2^{p_{+}}} W_{p(.)}^{p_{+}}\left(\rho_{1}, \rho_{2}\right) \\
& \quad \leq \int_{\Omega}<x-T^{+}, \nabla_{x}\left(G^{\prime}\left(\rho_{1}\right)+V\right)>\rho_{1} \mathrm{~d} x .
\end{aligned}
$$

In particular, if $\rho_{1}=\rho_{\infty} \in P(\Omega) \quad$ satisfies $\rho_{\infty} \nabla_{x}\left(G^{\prime}\left(\rho_{\infty}\right)+V\right)=0$ and $\rho_{2}=\rho \in P(\Omega)$, then

$$
E\left(\rho_{\infty}\right)-E(\rho)+\frac{\lambda}{2^{p_{+}}} W_{p(.)}^{p_{+}}\left(\rho_{\infty}, \rho\right) \leq 0 .
$$

So, we have

$$
W_{p(.)}^{p_{+}}\left(\rho_{\infty}, \rho\right) \leq \frac{2^{p_{+}}}{\lambda}\left[E(\rho)-E\left(\rho_{\infty}\right)\right],
$$

for all $\rho \in P(\Omega)$.

Note that $\rho_{\infty}$ is a stationary solution of the $q(x)$-Laplacian parabolic equation, which minimizes the functional $E(\rho)=\int_{\Omega}(G(\rho)+\rho V) \mathrm{d} x$ on $P(\Omega)$.

Lemma 5. Let $\rho_{1}, \rho_{2} \in P(\Omega)$. Then,

$$
E\left(\rho_{1}\right)-E\left(\rho_{2}\right) \leq \frac{2^{p_{+1}}}{\lambda^{p_{+1}}}\left\|\nabla_{x}\left(G^{\prime}\left(\rho_{1}\right)+V\right)\right\|_{L_{\rho_{1}}^{q(.)}(\Omega)^{\prime}}^{p_{+}^{\prime}}
$$

with $p_{+}^{\prime}=\left(p_{+} /\left(p_{+}-1\right)\right)$.

Proof 8 . We applied Young's inequality to obtain

$$
<x-T_{+}, \nabla_{x}\left(G^{\prime}\left(\rho_{1}\right)+V\right)>\leq \mu\left|x-T_{+}\right|^{p_{+}}+\frac{1}{\mu^{p_{+}}}\left|\nabla_{x}\left(G^{\prime}\left(\rho_{1}\right)+V\right)\right|^{p_{+1}},
$$

where $\mu>0$ and $p_{+}^{\prime}=\left(p_{+} /\left(p_{+}-1\right)\right)$. Then,

$$
\int_{\Omega}<x-T_{+}, \nabla_{x}\left(G^{\prime}\left(\rho_{1}\right)+V\right)>\rho_{1} \mathrm{~d} x \leq \mu \int_{\Omega}\left|x-T_{+}\right|^{p_{+}} \rho_{1} \mathrm{~d} x+\frac{1}{\mu^{p_{+}}} \int_{\Omega}\left|\nabla_{x}\left(G^{\prime}\left(\rho_{1}\right)+V\right)\right|^{p_{+1}} \rho_{1} \mathrm{~d} x,
$$

and since $p_{1^{+}}^{\prime} \leq q(x)$, we use the continuous injection $L_{\rho_{1}}^{q(.)}(\Omega) \hookrightarrow L_{\rho_{1}}^{P_{+}}(\Omega)$, and we get

$\int_{\Omega}\left|\nabla_{x}\left(G^{\prime}\left(\rho_{1}\right)+V\right)\right|^{p_{+1}} \rho_{1} \mathrm{~d} x \leq 2^{p_{+1}}\left\|\nabla_{x}\left(G^{\prime}\left(\rho_{1}\right)+V\right)\right\|_{L_{\rho_{1}}^{q(.)}(\Omega)}^{p^{\prime}}$. 
Now, we combine (128), (137), and (136) to obtain

$$
\begin{gathered}
E\left(\rho_{1}\right)-E\left(\rho_{2}\right)+(\lambda-\mu) W_{p_{+}}^{p_{+}}\left(\rho_{1}, \rho_{2}\right) \\
\leq \frac{2^{p_{+}^{\prime}}}{\mu^{p_{+}^{\prime}}}\left\|\nabla_{x}\left(G^{\prime}\left(\rho_{1}\right)+V\right)\right\|_{L_{\rho_{1}}^{q(.)}(\Omega)}^{p_{+}^{\prime}} .
\end{gathered}
$$

In particular, if $\mu=\lambda$, we obtain (135).

\section{Lemma 6.}

$$
\left\|\nabla_{x}\left(G^{\prime}\left(\rho_{1}\right)+V\right)\right\|_{L_{\rho_{1}}^{q(.)}(\Omega)}^{p_{+}^{\prime}} \leq \operatorname{Max}\left\{\int_{\Omega}\left|\nabla_{x}\left(G^{\prime}\left(\rho_{1}\right)+V\right)\right|^{q(x)} \rho_{1} \mathrm{~d} x,\left(\int_{\Omega} \nabla_{x}\left(G^{\prime}\left(\rho_{1}\right)+V\right)^{q(x)} \rho_{1} \mathrm{~d} x\right)^{\left(1 / q_{+}\right)}\right\} .
$$

Proof 9. From [9], we get

$$
\left\|\nabla_{x}\left(G^{\prime}\left(\rho_{1}\right)+V\right)\right\|_{L_{\rho_{1}}^{q(.)}(\Omega)}^{q_{-}} \leq \int_{\Omega}\left|\nabla_{x}\left(G^{\prime}\left(\rho_{1}\right)+V\right)\right|^{q(x)} \rho_{1} \mathrm{~d} x, \quad \text { if }\left\|\nabla_{x}\left(G^{\prime}\left(\rho_{1}\right)+V\right)\right\|_{L_{\rho_{1}}^{q(.)}(\Omega)} \geq 1
$$

and

$$
\left\|\nabla_{x}\left(G^{\prime}\left(\rho_{1}\right)+V\right)\right\|_{L_{\rho_{1}}^{q(.)}(\Omega)}^{p_{+}^{\prime}} \leq \int_{\Omega}\left|\nabla_{x}\left(G^{\prime}\left(\rho_{1}\right)+V\right)\right|^{q(x)} \rho_{1} \mathrm{~d} x, \quad \text { if }\left\|\nabla_{x}\left(G^{\prime}\left(\rho_{1}\right)+V\right)\right\|_{L_{\rho_{1}}^{q(.)}(\Omega)}<1
$$

Since $1<p_{+}^{\prime} \leq q_{-}$, we have

$$
\begin{gathered}
\left\|\nabla_{x}\left(G^{\prime}\left(\rho_{1}\right)+V\right)\right\|_{L_{\rho_{1}}^{q(.)}(\Omega)}^{p_{+}^{\prime}} \leq\left|\nabla_{x}\left(G^{\prime}\left(\rho_{1}\right)+V\right)\right|^{q(x)} \rho_{1} \mathrm{~d} x, \quad \text { if }\left\|\nabla_{x}\left(G^{\prime}\left(\rho_{1}\right)+V\right)\right\|_{L_{\rho_{1}}^{q(.)}(\Omega)} \geq 1, \\
\left\|\nabla_{x}\left(G\left(\rho_{1}\right)+V\right)\right\|_{L_{\rho_{1}}^{q(.)}(\Omega)}^{p_{+}^{\prime}} \leq\left\|\nabla_{x}\left(G^{\prime}\left(\rho_{1}\right)+V\right)\right\|_{L_{\rho_{1}}^{q(.)}(\Omega)}, \quad \text { if }\left\|\nabla_{x}\left(G^{\prime}\left(\rho_{1}\right)+V\right)\right\|_{L_{\rho_{1}}^{q(.)}(\Omega)}<1 .
\end{gathered}
$$

By using (141), we derive that

$$
\left\|\nabla_{x}\left(G^{\prime}\left(\rho_{1}\right)+V\right)\right\|_{L_{\rho_{1}}^{q(.)}(\Omega)} \leq\left(\int_{\Omega}\left|\nabla_{x}\left(G^{\prime}\left(\rho_{1}\right)+V\right)\right|^{q(x)} \rho_{1} \mathrm{~d} x\right)^{\left(1 / q_{+}\right)}, \quad \text { if }\left\|\nabla_{x}\left(G^{\prime}\left(\rho_{1}\right)+V\right)\right\|_{L_{\rho_{1}}^{q(.)}(\Omega)}<1 .
$$

Next, we combine (143) and (144) to derive

$$
\left\|\nabla_{x}\left(G^{\prime}\left(\rho_{1}\right)+V\right)\right\|_{L_{\rho_{1}}^{q(.)}(\Omega)}^{p^{\prime}} \leq\left(\int_{\Omega}\left|\nabla_{x}\left(G^{\prime}\left(\rho_{1}\right)+V\right)\right|^{q(x)} \rho_{1} \mathrm{~d} x\right)^{\left(1 / q_{+}\right)}, \quad \text { if }\left\|\nabla_{x}\left(G^{\prime}\left(\rho_{1}\right)+V\right)\right\|_{L_{\rho_{1}}^{q(.)}(\Omega)}<1 .
$$

By using (142) and (143), we obtain (140).
4.1. Proof of Theorem 2. Let $\rho(t, x)$ be a solution of the $q(x)$ Laplacian parabolic equation. We have 


$$
\frac{d}{\mathrm{~d} t}\left[E(\rho(t, .))-E\left(\rho_{\infty}\right)\right]=-\int_{\Omega}\left|\nabla_{x}\left(G^{\prime}(\rho(t, x))+V\right)\right|^{q(x)} \rho(t, x) \mathrm{d} x
$$

Define

$$
\frac{d}{\mathrm{~d} t}\left[E(\rho(t, .))-E\left(\rho_{\infty}\right)\right] \leq-\left(\frac{\lambda}{2}\right)^{p_{+} \prime}\left[E(\rho(t, .))-E\left(\rho_{\infty}\right)\right]
$$

$I_{1}:=\left\{t \in\left[0,+\infty\left[, \quad\left\|\nabla_{x}\left(G^{\prime}(\rho(t, .))+V\right)\right\|_{L_{\rho(t, .)}^{q(.)}(\Omega)} \geq 1\right\}\right.\right.$,

$I_{2}:=\left\{t \in\left[0,+\infty\left[, \quad\left\|\nabla_{x}\left(G^{\prime}(\rho(t, .))+V\right)\right\|_{L_{\rho(t, .)}^{q(.)}(\Omega)}<1\right\}\right.\right.$.

on $I_{1}$, and we combine (135), (145), and (146) to have

$$
\frac{d}{\mathrm{~d} t}\left[E(\rho(t, .))-E\left(\rho_{\infty}\right)\right] \leq-\left(\frac{\lambda}{2}\right)^{p_{+} q_{+}}\left[E(\rho(t, .))-E\left(\rho_{\infty}\right)\right]^{q_{+}}
$$

We combine (135), (142), and (146) to get

on $I_{2}$.

Next, we have after integration,

$$
\left.\left[E(\rho(t, .))-E\left(\rho_{\infty}\right)\right] \leq \frac{\left[E\left(\rho\left(t_{2}, .\right)\right)-E\left(\rho_{\infty}\right)\right]}{\left[1+(\lambda / 2)^{p_{+} q_{+}}\left(q_{+}-1\right)\left(E\left(\rho\left(t_{2}, .\right)\right)-E\left(\rho_{\infty}\right)\right)\left(q_{+}-1\right)\right.}\left(t-t_{2}\right)\right]^{\left(1 / q_{+}-1\right)},
$$

on $I_{2}$, and

$$
\left.\left[E(\rho(t, .))-E\left(\rho_{\infty}\right)\right]\right] \leq e^{-(\lambda / 2)^{p_{+}}}\left(t-t_{1}\right)\left[E\left(\rho_{0}\right)-E\left(\rho_{\infty}\right)\right] \text {, }
$$

on $I_{1}$.

To conclude, we use Lemma 4 and expressions (151) and (152), and then, the proof of Theorem 2 is complete.

\section{Error Estimate}

In this section, we provide in the $L^{2}$ norm an estimate of the gap between the approximate solution and the exact solution.
Lemma 7. Assume that $\left(H_{\rho_{0}}\right),\left(H_{G}\right),\left(H_{q(.)}^{p(.)}\right)$, and $\left(H_{V}^{1}\right)$ hold. Let $\rho=\rho(t, x)$ be a weak solution of the $q(x)$-Laplacian parabolic equation and $\rho^{h}=\rho^{h}(t, x)$ be an approximate solution of the $q(x)$-Laplacian parabolic equation defined in (12). Then,

$$
\left\|\rho-\rho^{h}\right\|_{L^{2}([0, T] \times \Omega)}^{2} \leq C(T, N, M, \rho, \Omega, G, p(.), q(.)) h,
$$

where $C(T, N, M, \rho, \Omega, G, p(),. q()$.$) is a constant depending$ on $T, N, M, \rho, \Omega, G, p(),. q($.$) .$

Proof 10. Since $\rho=\rho(t, x)$ is a weak solution of the $q(x)$ Laplacian parabolic equation, then

$$
\rho(t, x)=\rho_{k-1}(x)+\int_{h(k-1)}^{t} \operatorname{div}_{x}(\rho)\left|\nabla_{x}\left(G^{\prime}(\rho)+V\right)\right|^{q(x)-2} \nabla_{x}\left(G^{\prime}(\rho)+V\right) \mathrm{d} s, \quad \text { for } t \in h(k-1), \text { hk. }
$$

Similarly, the approximate solution $\rho^{h}$ satisfies

$$
\rho^{h}(t, x)=\rho_{k-1}(x)+\int_{h(k-1)}^{t} \operatorname{div}_{x}\left(\rho^{h}\right)\left|\nabla_{x}\left(G^{\prime}\left(\rho^{h}\right)+V\right)\right|^{q(x)-2} \nabla_{x}\left(G^{\prime}\left(\rho^{h}\right)+V\right) \mathrm{d} s, \quad \text { for } t \in h(k-1), \mathrm{hk}
$$

We combine (154) and (155) to have

$$
\begin{aligned}
& \rho(t, x)-\rho^{h}(t, x)= \\
& \int_{h(k-1)}^{t}\left[\left(\operatorname{div}_{x}\left(\rho\left|\nabla_{x}\left(G^{\prime}(\rho)+V\right)\right|^{q(x)-2} \nabla_{x}\left(G^{\prime}(\rho)+V\right)\right)-\operatorname{div}_{x}\left(\rho^{h}\left|\nabla_{x}\left(G^{\prime}\left(\rho^{h}\right)+V\right)\right|^{q(x)-2} \nabla_{x}\left(G^{\prime}\left(\rho^{h}\right)+V\right)\right)\right)\right] \mathrm{d} s .
\end{aligned}
$$


Discrete Dynamics in Nature and Society

19

We multiply (156) by $G^{\prime}(\rho)-G^{\prime}\left(\rho^{h}\right)$, and we integrate it on $[0, T] \times \Omega$. Consequently,

$$
\int_{[0, T] \times \Omega}\left[\left[\rho(t, x)-\rho^{h}(t, x)\right]\left[G^{\prime}(\rho)-G^{\prime}\left(\rho^{h}\right)\right]\right] \mathrm{d} t \mathrm{~d} x=-\int_{[0, T] \times \Omega} \int_{h(k-1)}^{t}<\rho|\sigma|^{q(x)-2} \sigma-\rho^{h}\left|\sigma^{h}\right|^{q(x)-2} \sigma^{h}, \sigma-\sigma^{h}>d t \mathrm{~d} x \mathrm{~d} s,
$$

where $\sigma=\nabla_{x}\left(G^{\prime}(\rho)+V\right)$ and $\sigma^{h}=\nabla_{x}\left(G^{\prime}\left(\rho^{h}\right)+V\right)$.

Since $N \leq \rho \leq M, N \leq \rho^{h} \leq M$, and $G$ is a convex function of class $C^{2}$, then $G^{\prime \prime} \geq 0$, and

$$
\begin{aligned}
& {\left[\rho(t, x)-\rho^{h}(t, x)\right]\left[G^{\prime}(\rho)-G^{\prime}\left(\rho^{h}\right)\right]} \\
& \quad=G^{\prime \prime}\left(\rho^{h}+\theta\left(\rho-\rho^{h}\right)\right)\left|\rho-\rho^{h}\right|^{2} \\
& \quad \geq k_{0}\left|\rho-\rho^{h}\right|^{2},
\end{aligned}
$$

$$
\int_{[0, T] \times \Omega}\left|\rho(t, x)-\rho^{h}(t, x)\right|^{2} \mathrm{~d} t \mathrm{~d} x \leq-\frac{1}{k_{0}}\left[\int_{[0, T] \times \Omega} \int_{h(k-1)}^{t}<\rho|\sigma|^{q(x)-2} \sigma-\rho^{h}\left|\sigma^{h}\right|^{q(x)-2} \sigma^{h}, \sigma-\sigma^{h}>\mathrm{d} t \mathrm{~d} x \mathrm{~d} s\right] .
$$

Since $N \leq \rho \leq M$ and $N \leq \rho^{h} \leq M$, then

$$
\begin{aligned}
& -\frac{1}{k_{0}}\left[\int_{[0, T] \times \Omega} \int_{h(k-1)}^{t}<\rho|\sigma|^{q(x)-2} \sigma-\rho^{h}\left|\sigma^{h}\right|^{q(x)-2} \sigma^{h}, \sigma-\sigma^{h}>\mathrm{d} t \mathrm{~d} x \mathrm{~d} s\right] \\
& \leq \frac{2 M}{k_{0}} h\left[\left\||\sigma|^{q(x)-2} \sigma\right\|_{L^{p(.)}([0, T] \times \Omega)}+\left\|\left|\sigma^{h}\right|^{q(x)-2} \sigma^{h}\right\|_{L^{p(.)}([0, T] \times \Omega)}\right]\left[\|\sigma\|_{L^{q(.)}([0, T] \times \Omega)}+\left\|\sigma^{h}\right\|_{L^{q(.)}([0, T] \times \Omega)}\right] .
\end{aligned}
$$

By using $\sigma=\nabla_{x}\left(G^{\prime}(\rho)+V\right)$ and $\sigma^{h}=\nabla_{x}\left(G^{\prime}\left(\rho^{h}\right)+V\right)$, we obtain

$$
\begin{gathered}
\left\|\sigma^{h}\right\|_{L^{q(.)}([0, T] \times \Omega)} \leq \max \left\{\left(\int_{[0, T] \times \Omega}\left|\nabla_{x}\left(G^{\prime}\left(\rho^{h}\right)+V\right)\right|^{q(x)} \mathrm{d} t \mathrm{~d} x\right)^{\left(1 / q_{+}\right)},\left(\int_{[0, T] \times \Omega}\left|\nabla_{x}\left(G^{\prime}\left(\rho^{h}\right)+V\right)\right|^{q(x)} \mathrm{d} t \mathrm{~d} x\right)^{\left(1 / q_{-}\right)}\right\}, \quad(161) \\
\left\|\left|\sigma^{h}\right|^{q(x)-2} \sigma^{h}\right\|_{L^{p(.)}([0, T] \times \Omega)} \leq \max \left\{\left(\int_{[0, T] \times \Omega}\left|\nabla_{x}\left(G^{\prime}\left(\rho^{h}\right)+V\right)\right|^{q(x)} \mathrm{d} x \mathrm{~d} t\right)^{\left(1 / p_{+}\right)},\left(\int_{[0, T] \times \Omega}\left|\nabla_{x}\left(G^{\prime}\left(\rho^{h}\right)+V\right)\right|^{q(x)} \mathrm{d} t \mathrm{~d} x\right)^{\left(1 / p_{-}\right)}\right\} .
\end{gathered}
$$

By using (64), then

$$
\int_{[0, T] \times \Omega}\left|\nabla_{x}\left(G^{\prime}\left(\rho^{h}\right)+V\right)\right|^{q(x)} \mathrm{d} t \mathrm{~d} x \leq \frac{\mathrm{Tp}_{+}}{N}\left[\int_{\Omega} G\left(\rho_{0}\right) \mathrm{d} x-|\Omega| G\left(\frac{1}{|\Omega|}\right)+2\|V\|_{L^{\infty}(\Omega)}\right] .
$$


Finally, we combine (159)-(163) to obtain the desired estimate:

$$
\begin{aligned}
& \int_{[0, T] \times \Omega}\left|\rho(t, x)-\rho^{h}(t, x)\right|^{2} \mathrm{~d} t \mathrm{~d} x \\
& \quad \leq C(T, N, M, \Omega, G, p(.), q(.)) h,
\end{aligned}
$$

where $C(T, N, M, \Omega, G, p(),. q()$.$) is a constant depending$ on $T, N, M, \Omega, G, p(),. q($.$) .$

\section{Numerical Example}

Here, we give an illustration of our work by testing the algorithm on an example of the $q(x)$-Laplacian parabolic equation in the dimension equal to 1 for determining an approximate solution. We limit ourselves to the first-order approximation just for the sake of simplicity and to avoid cumbersome calculations. An error table is obtained from different values of step $h$, showing that the error values decrease to zero in the $L^{2}$-norm when $h$ tends to zero, in relevance with the similarity between the graphical representations of both the analytical solution and the approximate solution.

$q(x)$-Laplacian parabolic equation is a nonhomogeneous equation and may be considered as a model of response over the time of nonhomogeneous media under the stress of a physical phenomenon. We suppose in our example that the functions $p, V$, and $G$ in the $q(x)$-Laplacian parabolic equation are as follows:

$$
\begin{aligned}
& V(x)=\frac{x^{2}}{2} \chi_{[(1 / 2),(3 / 4))}, \quad \text { if } x \in(0,1), \\
& q(x)=\frac{5}{2} \chi_{(0,(1 / 2)) \cup[(3 / 4), 1)}+2 \chi_{[(1 / 2),(3 / 4))}, \\
& G(x)=\frac{9}{4} x^{\frac{3}{4}} \chi_{(0,(1 / 2)) \cup[(3 / 4), 1)}+x \ln (x) \chi_{[(1 / 2),(3 / 4))}, \quad \text { for } x \in(0,1) .
\end{aligned}
$$

Consequently, the parabolic $q(x)$-Laplacian parabolic equation becomes the following couple of the linear Fokker-Planck equation and the (5/2)-Laplacian parabolic equation:

$$
\left\{\begin{aligned}
\frac{\partial \rho}{\partial t} & =\Delta_{x} \rho+\operatorname{div}_{x}(x \rho) \text { in }[0,+\infty) \times\left[\frac{1}{2}, \frac{3}{4}\right) \\
\frac{\partial \rho}{\partial t} & =\operatorname{div}_{x}\left(\left|\nabla_{x} \rho\right|^{(1 / 2)} \nabla_{x} \rho\right) \text { in }[0,+\infty) \\
& \times\left(0, \frac{1}{2}\right) \cup\left[0,+\infty\left[\times\left[\frac{3}{4}, 1\right) .\right.\right.
\end{aligned}\right.
$$

One can assimilate the set $(0,1)$ to a nonhomogeneous composite rod of length 1 cast in two distinct materials and made of a main piece $[(1 / 2),(3 / 4))$ surrounded at its extremities by two other pieces $(0,(1 / 2))$ and $[(3 / 4), 1)$. The nonhomogeneity is characterized by the two different constant values taken by the variable exponent $q(x)$ on the distinct pieces of the rod.
Let us express the solution of the linear Fokker-Planck equation

$$
\frac{\partial \rho}{\partial t}=\Delta_{x} \rho+\operatorname{div}_{x}(x \rho)
$$

in the form

$$
\begin{aligned}
\rho(t, x) & =e^{t} u(\tau, y), \\
(\tau, y) & =\left(\frac{1}{2} e^{2 t}, e^{t} x\right),
\end{aligned}
$$

where $u(\tau, y)$ is an unknown function, supposed to be a solution of the heat equation

$$
\frac{\partial u}{\partial \tau}=\Delta_{y} u
$$

Thus, after a simple computation, we have that

$$
\begin{aligned}
\frac{\partial \rho}{\partial t} & =e^{t} u(\tau, y)+e^{3 t} \frac{\partial u}{\partial \tau}+x e^{2 t} \frac{\partial u}{\partial y}, \\
\Delta_{x} \rho & =e^{3 t} \Delta_{y} u, \\
\operatorname{div}_{x}(x \rho) & =e^{t} u(\tau, y)+x e^{2 t} \frac{\partial u}{\partial y} .
\end{aligned}
$$

So, if $u=u(t, x)$ satisfies

$$
\frac{\partial u}{\partial \tau}=\Delta_{y} u
$$

then $\rho(t, x)=e^{t} u(\tau, y)$ satisfies

$$
\frac{\partial \rho}{\partial t}=\Delta_{x} \rho+\operatorname{div}_{x}(x \rho)
$$

Besides, the function $u(\tau, y)=(1 / \sqrt{4 \tau+1}) e^{\left(-y^{2} / 4 \tau+1\right)}$ is an exact solution of heat equation (169). Accordingly, the function $\rho(t, x)=e^{t} u\left((1 / 2) e^{2 t}, e^{t} x\right)$ defined by

$$
\rho(t, x)=\frac{e^{t}}{\sqrt{2 e^{2 t}+1}} e^{-\left(e^{2 t} x^{2} / 2 e^{2 t}+1\right)},
$$

is an exact solution of equation (167).

Next, let us deal with the parabolic 5/2-Laplacian equation:

$$
\frac{\partial \rho}{\partial t}=\operatorname{div}_{x}\left(\left(\left|\nabla_{x} \rho\right|\right)^{(1 / 2)} \nabla_{x} \rho\right) .
$$
form

We obtain an exact solution $\rho=\rho(t, x)$ of (174) in the

$$
\rho(t, x)=\frac{4 x^{5}}{5(30 t+1)^{2}} .
$$

Finally, fix the initial datum $\rho_{0}$ to be

$$
\begin{cases}\rho_{0}(x)=\frac{4}{5} x^{5}, & \text { if } x \in\left(0, \frac{1}{2}\right) \cup\left[\frac{3}{4}, 1\right), \\ \rho_{0}(x)=\frac{1}{\sqrt{3}} e^{-\left(x^{2} / 3\right)}, & \text { if } x \in\left[\frac{1}{2}, \frac{3}{4}\right) .\end{cases}
$$




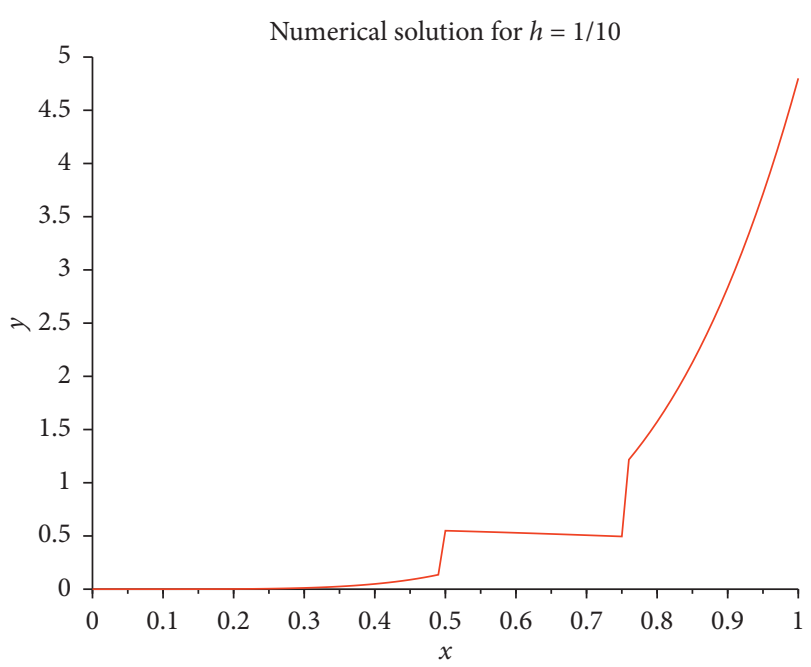

FIGURE 1: Numerical solution $\rho_{1}^{(1 / 10)}(x)$ for $h=(1 / 10)$.

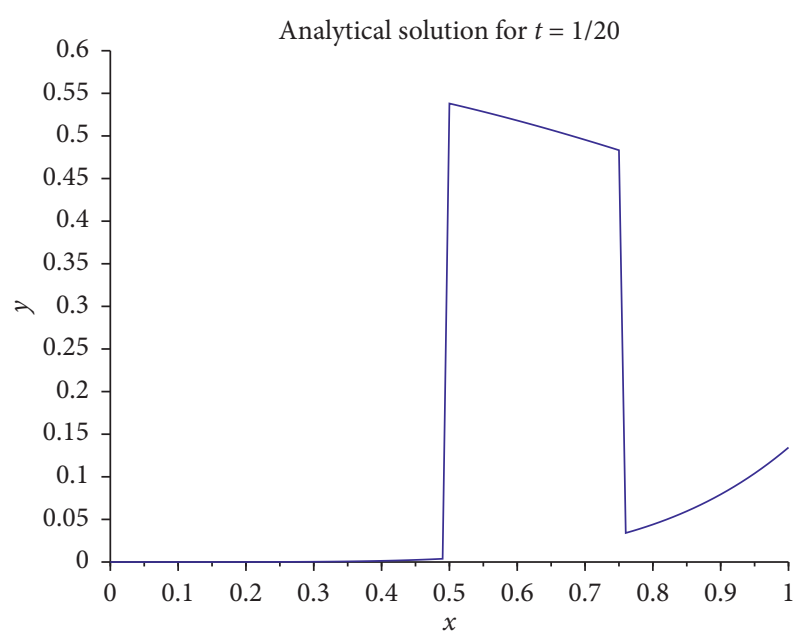

Figure 2: Analytical solution $\rho((1 / 20), x)$ for $h=(1 / 10)$ and $t=(1 / 20) \in(0,(1 / 10)]$.

Then, the exact solution $\rho=\rho(t, x)$ of the $q(x)$-Laplacian parabolic equation is presented as follows:

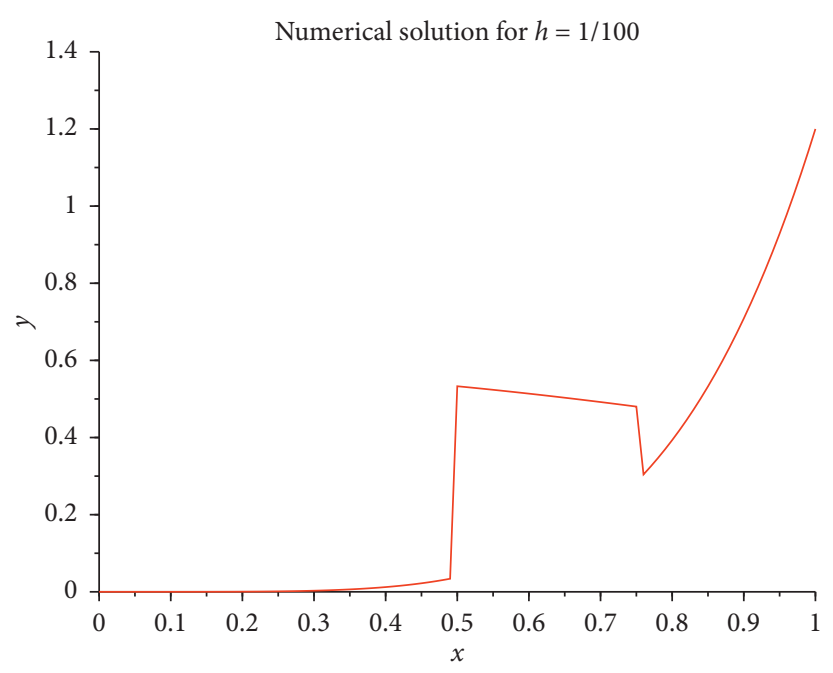

FIGURE 3: Numerical solution $\rho^{(1 / 1000)}(x)$ for $h=(1 / 100)$.

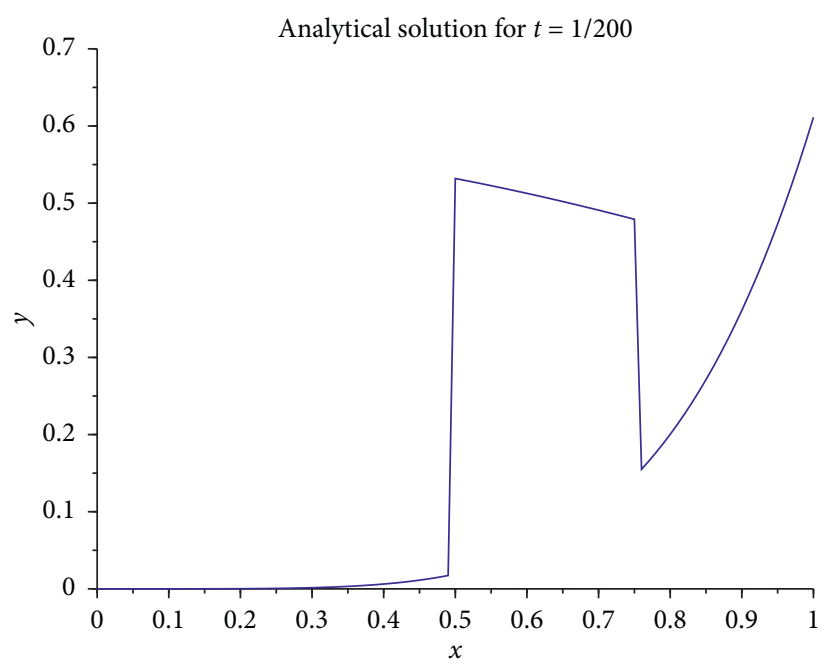

FIgURE 4: Analytical solution $\rho((1 / 200), x)$ for $h=(1 / 100)$ and $t=(1 / 2000) \in(0,(1 / 1000)]$.

$$
\begin{cases}\rho(t, x)=\frac{4 x^{5}}{5(30 t+1)^{2}}, & \text { if }(t, x) \in[0,+\infty) \times\left(0, \frac{1}{2}\right) \cup\left[0,+\infty\left[\times\left[\frac{3}{4}, 1\right),\right.\right. \\ \rho(t, x)=\frac{e^{t}}{\sqrt{2 e^{2 t}+1}} e^{-\left(e^{2 t} x^{2} / 2 e^{2 t}+1\right)}, & \text { if }(t, x) \in[0,+\infty) \times\left[\frac{1}{2}, \frac{3}{4}\right) .\end{cases}
$$

In this part of our work, we are interested in the existence of the stationary solution for our example. With the functions $G$ and $V$ as defined in the above, we deduce that the equation

$$
\rho_{\infty} \nabla_{x}\left(G^{\prime}\left(\rho_{\infty}\right)+V\right)=0
$$

becomes 


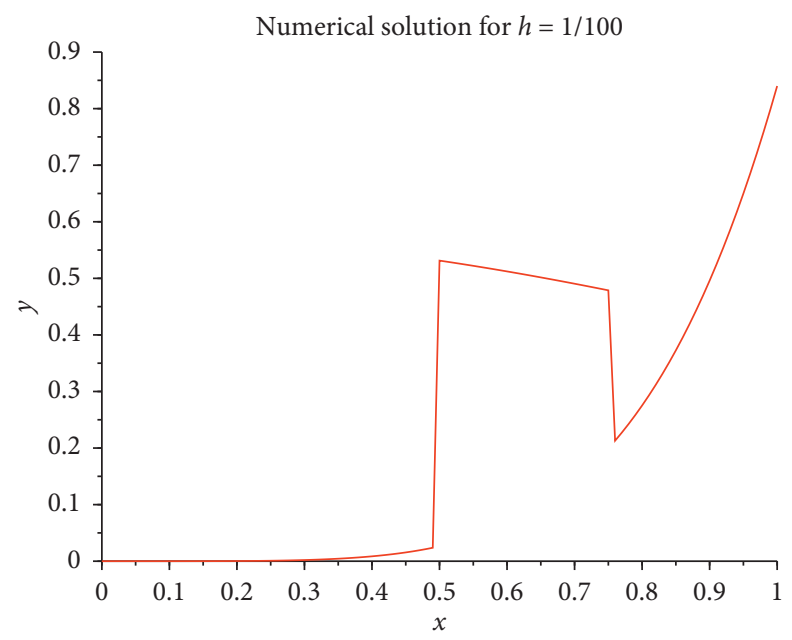

Figure 5: Numerical solution $\rho^{(1 / 1000)}(x)$ for $h=(1 / 1000)$.

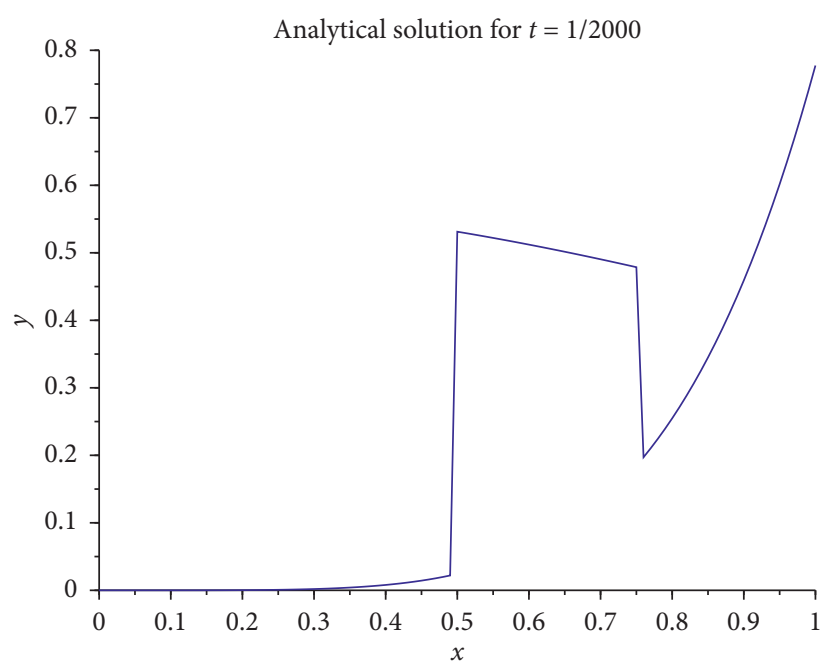

Figure 6: Analytical solution $\rho((1 / 2000), x)$ for $h=(1 / 1000)$ and $t=(1 / 2000) \in(0,(1 / 1000)]$.

TABle 1: Error table.

\begin{tabular}{lccccccc}
\hline$h$ & $1 / 10$ & $1 / 50$ & $1 / 100$ & $1 / 500$ & $1 / 1000$ & $1 / 5000$ & $1 / 10000$ \\
\hline$\delta t$ & $1 / 20$ & $1 / 100$ & $1 / 200$ & $1 / 1000$ & $1 / 2000$ & $1 / 20000$ & $1 / 20000$ \\
$\left\|\rho-\rho^{h}\right\|_{L^{2}}$ & 14.144343 & 3.3886130 & 1.7847695 & 0.3764119 & 0.1896313 & 0.0381622 & 0.0190961 \\
\hline
\end{tabular}

$$
\left\{\begin{array} { l l } 
{ \rho _ { \infty } \nabla _ { x } ( \rho _ { \infty } ^ { ( 1 / 3 ) } ) = 0 , } & { \text { in } ( 0 , \frac { 1 } { 2 } ) \cup [ \frac { 3 } { 4 } , 1 ) , } \\
{ \rho _ { \infty } \nabla _ { x } ( 1 + \operatorname { l n } ( \rho _ { \infty } ) + \frac { x ^ { 2 } } { 2 } ) = 0 , } & { \text { in } [ \frac { 1 } { 2 } , \frac { 3 } { 4 } ) . }
\end{array} \left\{\begin{array}{ll}
\rho_{\infty}(x)=0, & \text { if } x \in\left(0, \frac{1}{2}\right) \cup\left[\frac{3}{4}, 1\right), \\
\rho_{\infty}=\frac{1}{\sqrt{2}} e^{-x^{2} / 2}, & \text { if } x \in\left[\frac{1}{2}, \frac{3}{4}\right)
\end{array}\right.\right.
$$

Consequently, the function $\rho_{\infty}$ which is defined by solves (179) as the stationary solution of the $q(x)$-Laplacian parabolic equation. 
In order to appreciate the effectiveness of our approximation method, we apply descent algorithm (6) to derive the approximate solution $\rho^{h}$ for comparison with the exact solution, and we get

$$
\begin{cases}\rho^{h}(t, x)=\frac{4}{5}(1+50 h) x^{5}, & \text { if }(t, x) \in(0, h] \times\left(0, \frac{1}{2}\right) \cup(0, h] \times\left[\frac{3}{4}, 1\right) \\ \rho^{h}(t, x)=\frac{\sqrt{3}}{3-h} e^{-\left(x^{2} / 3\right)}, & \text { if }(t, x) \in(0, h] \times\left[\frac{1}{2}, \frac{3}{4}\right) .\end{cases}
$$

6.1. The Figures. For some values of $h$, we have drawn the figures of both analytical and approximate solutions when step $h \in\{(1 / 10),(1 / 50),(1 / 100)\}$, using Scilab software. We mention that Figure 1 represents an approximation of Figure 2 when, Figure 3 represents an approximation of Figure 4 when $h=(1 / 100)$, and Figure 5 represents an approximation of Figure 6 when $h=(1 / 1000)$.

6.2. Table of Error Progression. The Table of Error Progression is provide in the Table 1

\section{Data Availability}

The data used to support the findings of this study are available from the corresponding author upon request.

\section{Conflicts of Interest}

The authors declare that they have no conflicts of interest.

\section{Acknowledgments}

This work was supported by IMSP under the CEA-SMA project grant.

\section{References}

[1] H. Zhan, "The stability of evolutionary p (x)-Laplacian equation," Zhan Boundary value Problems, vol. 13, 2017.

[2] S. Lian, W. Gao, H. Yuan, and C. Cao, "Existence of solutions to an initial Dirichlet problem of evolutional p (x)-Laplace equations," Annales de l'Institut Henri Poincare (C) Non Linear Analysis, vol. 29, no. 3, pp. 377-399, 2012.

[3] Y. Chen, S. Levine, and M. Rao, "Variable exponent, linear growth functionals in image restoration," SIAM Journal on Applied Mathematics, vol. 66, no. 4, pp. 1383-1406, 2006.

[4] S. Antontsev, F. Miranda, and L. Santos, "Blow-up and finite time extinction for $\mathrm{p}(\mathrm{x}, \mathrm{t})$-curl systems arising in electromagnetism," Journal of Mathematical Analysis and Applications, vol. 440, no. 1, pp. 300-322, 2016.

[5] A. Bahrouni and D. Repovš, "Existence and nonexistence of solutions for $\mathrm{p}(x)$-curl systems arising in electromagnetism," Complex Variables and Elliptic Equations, vol. 63, no. 2, pp. 292-301, 2018.

[6] T. G. Myers, "Thin films with high surface tension," SiAM Review, vol. 40, no. 3, pp. 441-462, 1998.

[7] M. Agueh, "Existence of solutions to degenerate parabolic equations via the Monge-Kantorovich theory," Advances in Difference Equations, vol. 10, pp. 309-360, 2005.
[8] M. Agueh, Asymptotic Behavior for Doubly Degenerate Parabolic Equations, Department of Mathematics, The University of British Columbia, Vancouver, B. C. V6T 1Z2, Canada, 2003.

[9] Z. Fan, On The Spaces $L^{p(x)}(\Omega)$ and, $W^{m, p(x)}(\Omega)$. Department of Mathematics, Lanzhou University, Lanzhou, China, 2009.

[10] M. Hamta and B. Salad, Existence of Positive Solutions and its Asymptotic Behavior of $(P(x), q(x))$-laplacian Parabolic System, Licence MDPI, Basel, Switzerland, 2019.

[11] G. Yanchao, "Extinction and asymptotic behavior of solution for nonlinear parabolic equations with variable exponent of nonlinearity," Boundary Value Problems, vol. 164, 2013.

[12] L. Diening, P. Harjulehto, P. Hasto, and M. Růžička, "Lebesgue and sobolev spaces with variable exponents," Lecture Notes in Mathematics, Springr, Berlin, Germany, 2017. 